\title{
The swing voter's curse in social networks
}

\author{
Berno Buechel ${ }^{\mathrm{a}}$, Lydia Mechtenberg ${ }^{\mathrm{b}, *}$ \\ a University of Fribourg, Department of Economics, bd. de Pérolles 90, CH-1700 Fribourg, Switzerland \\ ${ }^{\mathrm{b}}$ University of Hamburg, Department of Economics, von-Melle-Park 5, D-20146 Hamburg, Germany
}

\section{A R T I C L E I N F O}

\section{Article history:}

Received 17 May 2018

Available online 3 September 2019

\section{JEL classification:}

D72

D83

D85

C91

\section{Keywords:}

Strategic voting

Social networks

Swing voter's curse

Information aggregation

\begin{abstract}
A B S T R A C T
We study communication in social networks prior to a majority vote on two alternative policies. Some agents receive a private imperfect signal about which policy is correct. They can recommend a policy to their neighbors in the social network prior to the vote. We show theoretically and empirically that communication can undermine efficiency and hence reduce welfare in a common-interest setting. Both efficiency and existence of fully informative equilibria in which vote recommendations are truthfully given and followed hinge on the structure of the network. If some voters have distinctly larger audiences than others, their neighbors should not follow their vote recommendation; however, they may do so in equilibrium. We test the model in a laboratory experiment and find rather inefficient equilibrium selection. Based on this result, there is support for the comparative statics of our model and, more generally, for the importance of the network structure for voting behavior.
\end{abstract}

(c) 2019 Elsevier Inc. All rights reserved.

\section{Introduction}

Motivation. Majority voting is a major form of collective decision making. As such, it is intensely studied in political economy. However, traditionally the literature has ignored pre-vote communication, although in reality, people often receive advice before they vote. For instance, family members, neighbors or Facebook friends who are more deeply interested in politics or better informed might try to convince others to vote like them. Vote recommendations seem to be frequent: Approximately $30 \%$ of the U.S. population report that they give vote recommendations to their peers often or sometimes (see Carpini et al., 2004, p. 323). Hence, the question arises whether pre-vote communication is harmless or even desirable, or whether such communication can be harmful. In the past two decades, there have been several theoretical and experimental

\footnotetext{
th We thank Marina Agranov, Francis Bloch, Jordi Brandts, Yann Bramoullé, Dirk Engelmann, Manuel Foerster, Matt Jackson, Dominik Karos, Shuo Liu, Friederike Mengel, and Jean-Robert Tyran for helpful comments. Moreover, we thank the participants of the conference on "Information Transmission in Networks" at Harvard University, the participants of the public economic theory (PET15) conference in Luxembourg, the Lisbon Meeting in Game Theory and Applications 2015, the SSES Annual Congress 2016 in Lugano, the Coalition Theory Network (CTN) workshop 2016 in Moscow, the SAET conference 2017 in Faro, and the Warwick/Princeton/Utah Political Economy Conference 2019 at Warwick in Venice, as well as the seminar participants at Caltech, in Cologne, Hamburg, Konstanz, Marseilles, Regensburg, Stanford and Zurich. We thank Willi Flemming for excellent research assistance. Berno Buechel gratefully acknowledges the hospitality of the Economics Department of Stanford University and the financial support by the Fritz Thyssen Foundation. Lydia Mechtenberg gratefully acknowledges the hospitality of the Institute for Economic Analysis (IAE) in Barcelona and the hospitality of Caltech in Pasadena.

* Corresponding author.

E-mail addresses: berno.buechel@unifr.ch (B. Buechel), Lydia.Mechtenberg@uni-hamburg.de (L. Mechtenberg).

URL: http://www.berno.info (B. Buechel).
} 
contributions to this issue, ranging from studying straw polls (Coughlan, 2000; Guarnaschelli et al., 2000) to studying all cheap-talk extensions of voting games (Gerardi and Yariv, 2007; Goeree and Yariv, 2011; Iaryczower et al., 2018). A central insight is that public communication among voters with sufficiently aligned preferences leads to efficient information aggregation due to deliberation of private signals (Coughlan, 2000, Austen-Smith and Feddersen, 2006, Gerardi and Yariv, 2007, and Goeree and Yariv, 2011). However, pre-vote communication need not be public, but can also be - at least partly private, as illustrated by the examples above. ${ }^{1}$

We focus our analysis on vote recommendations that are provided in one round of (partly) private communication. We show that this form of pre-vote communication can impede efficient information aggregation even if voters are sophisticated and interests are perfectly aligned. We restrict our analysis to a common-interest setting to take the assumption to the extreme that voters' preferences are sufficiently aligned to allow for truthful communication. Thus, we demonstrate a negative effect of communication that is, other than in the cheap-talk literature, not due to limited degrees of truthfulness or to conflicting interests, but rather to the exogenous structure of the communication network. Hence, our setting predominantly applies to large elections, involving a high degree of uncertainty, and concerning a "common good" like national security or growth or, in the case of shareholder votes, the future of the company in question. ${ }^{2}$

A negative effect of private pre-vote communication on efficiency can occur if the social network connecting voters who are (imperfect) experts on the issue at stake with other voters is not sufficiently balanced. In insufficiently balanced networks, some voters have a somehow larger audience than other voters without having much better information. In some such networks it is an equilibrium strategy to follow the vote recommendations one receives. Hence, wrongly informed voters may get too much weight in the vote. Then, the voting outcome is less efficient than it would have been in the absence of pre-vote communication. We show that this result is robust: It even holds true if the number of informed voters goes to infinity.

Since our theoretical analysis is restricted to two focal types of equilibria (one with giving and following vote recommendations and one with voting according to one's signal when informed and abstaining otherwise), we conducted a laboratory experiment to test our focality assumption and to investigate equilibrium selection in different networks. The second purpose of the experiment is to investigate how subjects account for the inefficiency mentioned above, in particular, whether uninformed voters follow vote recommendations of informed voters less often if the latter have an oversized audience. The laboratory data validate our focality assumption, with more coordination on giving and following vote recommendations than on voting according to one's signal when informed and abstaining otherwise. We find that individual behavior is largely in line with the comparative statics of our theory: Giving and following vote recommendations becomes less frequent when the network is unbalanced. On the collective level, there are more differences between our comparative statics and empirical group behavior, but our data reveal that in the laboratory, too, truthful communication sometimes impedes efficient information aggregation. ${ }^{3}$

To better understand when and why vote recommendations can be harmful and when they are harmless, consider communication networks in which imperfectly informed voters give vote recommendations to their neighbors. Such a communication stage is introduced into a standard common-interest voting game. Nature draws the binary state of the world and the signals that voters receive on the true state. Both states of the world are equally likely. Each informed voter receives only one signal, and signals are independent across voters. Some voters have audiences of one or more other voters and can send one out of two possible messages, which can be interpreted as vote recommendations, to their audience or keep silent. ${ }^{4}$ Then, a vote takes place to decide which of two possible policies shall be implemented. Only the policy matching the true state of the world generates a strictly positive payoff for all individuals (the other policy generates a zero payoff for everyone). Voters individually and simultaneously decide between voting for one or the other policy and abstaining. Voting is costless. ${ }^{5}$ The policy that gets a simple majority of votes is implemented. In case the voting outcome is a tie, the policy to be implemented is randomly drawn, where both policies have equal probability. Voters are strategic; i.e., they condition their behavior on pivotality.

A focal strategy in this setting is to recommend the policy indicated by one's own signal and to vote according to one's updated belief about the "correct" policy. We call this strategy sincere behavior and investigate when it is an equilibrium and when efficient. Consider an informed voter whose audience - consisting of her neighbors in the network - is a substantial part of the voting population, i.e., she is a well-connected sender. In a model of naïve social learning, her audience

\footnotetext{
1 Iaryczower et al. (2018) study all communication equilibria of a jury-voting game when jury members' utility functions have both common-interest and private-interest components. They find positive and negative effects of deliberative communication. In a setting of naïve learning among non-strategic voters, Golub and Jackson (2012) show that private communication in homophilous networks can undermine efficient information aggregation in the intermediate term. Outside the literature on information aggregation, Agranov et al. (2017) and Palfrey and Pogorelskiy (2017) study public and private pre-vote communication. In their settings, communication reveals information on preferences or intentions to turn out, which affects actual turnout.

2 In an extension, we show that our results are robust to the introduction of propaganda by voters with extreme biases ("partisans" or "trolls").

3 We also conducted a second laboratory experiment testing the same model, extended by partisans (or troll senders), with different social networks. Our findings from the second experiment, which we refer to as Study II, corroborate the picture painted from the main experiment. For further detail, see section C.3 in the supplementary online material (SOM).

4 In our set-up, the chosen message space is rich enough to convey all information.

5 With costs of voting, the pivot probability which might change across equilibria in different networks would affect the willingness to abstain. Since we want to isolate the effects of communication on voting behavior, we abstract from voting costs. In the laboratory, costless voting makes the "willingness to delegate to the expert" harder to find and hence more surprising.
} 
would simply follow this sender's vote recommendation, rendering her an opinion leader whose influence undermines efficient learning (e.g. DeMarzo et al., 2003; Golub and Jackson, 2010). In contrast, we consider behavior of Bayesian updaters who are strategic agents: Being pivotal with a vote that follows the well-connected sender's recommendation implies that many voters from the rest of the population voted for the opposite, which implies, in turn, that they had information contradicting the sender's recommendation. Hence, conditioning on pivotality, it is more likely that the vote recommendation of the sender is based on wrong rather than correct information. More generally, in unbalanced networks in which the power to influence opinions is insufficiently justified by the expertise of the sender, sincere behavior is neither informationally efficient nor equilibrium behavior. However, we state as a main result that there are communication networks (denoted as weakly balanced, but not strongly balanced) such that sincere behavior is both an equilibrium and informationally inefficient. In Appendix A.2, where we generalize our model to arbitrary networks, we prove that our results are robust and neither existence nor inefficiency vanish in the limit (when the network of voters grows ad indefinitum with the structure preserved).

An important feature of our model is that the exogenously given network structure only determines the system of communication channels that can potentially be used, while there is always an equilibrium without communication. Indeed, there is an alternative focal strategy: Voters who are better informed than others vote for the policy indicated by their signal and the others abstain. In line with the literature, we call this strategy "let the experts decide" (henceforth: LTED). ${ }^{6}$ Importantly, we show that LTED is efficient, independent of the network structure. Hence, in a class of weakly but not strongly balanced networks the problem of efficiency becomes one of equilibrium selection between sincere behavior and LTED, which is essentially an empirical question.

Testing our theoretical predictions in the laboratory experiment, we find that (i) collectively, groups switch more often from sincere to LTED if the network becomes unbalanced, but that (ii) sincere behavior still occurs frequently even in unbalanced networks, causing a (weak) loss in efficiency, compared to balanced networks. (iii) Individually, uninformed voters are indeed more inclined to abstain when they are in an unbalanced network (and hence listen to a powerful sender) and (iv) informed voters tend to pass on their signals to their audience but become more reluctant to do so when they are, in an unbalanced network, a sender with an oversized audience. In the experiment, there is loss in informational efficiency when the communication network becomes unbalanced. Intuitively, the less balanced the network structure, the less balanced is the power to influence opinions such that the final outcome is determined by the messages of a few agents, in contrast to the Marquis de Condorcet's original idea of aggregating information in the entire collective (M.d. Condorcet, 1785).

Related literature. Condorcet's argument that majority voting among independently informed voters efficiently aggregates private signals, i.e., his "Jury Theorem," is a cornerstone of the justification of the majority rule, and, even more generally, of making collective decisions by voting. His argument has been seriously challenged by Austen-Smith and Banks (1996) and Feddersen and Pesendorfer $(1996,1997,1998)$ who study voters as strategic actors. As they show, to vote in line with one's private information, i.e., to "sincerely" cast the vote for the alternative that maximizes unconditional expected utility, is not automatically an optimal action. When restricting attention to the cases in which one's own vote is decisive, the resulting conditional expected utility may be different. Hence, we assume strategic voting when solving our model, but also address the question when sincere and strategic voting lead to the same behavior.

In the absence of communication, Feddersen and Pesendorfer (1996) find that it is optimal for rational voters with common interests to abstain if they are uninformed and to vote in line with their independent private signal if they are informed. ${ }^{7,8}$ This LTED behavior not only forms an equilibrium, but also exhibits informational efficiency. In their experimental study of the model of Feddersen and Pesendorfer (1996), Battaglini et al. (2010) find that this equilibrium provides a good prediction of real behavior. Morton and Tyran (2011) have extended the model of Feddersen and Pesendorfer (1996) to include heterogeneity in information quality among the informed voters and find that less well-informed voters generally tend to abstain and delegate the collective decision to the better informed voters. Hence, the tendency to "delegate to the expert" seems quite strong in the laboratory. This suggests that the LTED equilibrium might be a good prediction even in more general models of information aggregation by majority votes. Accordingly, we consider it to be a benchmark equilibrium in our model, too.

However, efficiency of information aggregation hinges on the assumption that all participating voters enter the majority vote with independent private pieces of information - which is fulfilled in the standard model of common-interest voting. ${ }^{9}$ But the picture becomes more complicated when a mechanism is introduced that leads to correlated information among

\footnotetext{
6 See, e.g. Morton and Tyran (2011).

7 Since Feddersen's and Pesendorfer's ingenious contribution, the finding that uninformed voters in a common-interest setting are better off abstaining from the vote has been dubbed the swing voter's curse. More generally, a voter is "cursed" if his optimal strategy conditional on his pivotality differs from what he would deem optimal if he did not condition his strategy on being pivotal, i.e., what he would choose as a dictator. We adopt this way of speaking.

8 If one deviates from the assumption of common interests by introducing a number of "partisans" who always vote into a pre-specified direction, then abstention does no longer need to be the optimal strategy of the uninformed voters.

9 Levy and Razin (2015) provide a model on informed voting which includes heterogeneous preferences among voters, different sources of information for each voter and voters who neglect the correlation between their information sources. They show that correlation neglect may improve the informational efficiency of the vote since it makes voters put more weight on information than on the conflict of interest.
} 
voters, despite their private independent signals. Pre-vote communication is clearly one important source of correlation. ${ }^{10}$ Coughlan (2000) and Austen-Smith and Feddersen (2006) find that deliberation under the majority rule fosters efficiency when preferences are sufficiently aligned. Guarnaschelli et al. (2000) validate this insight experimentally taking Coughlan (2000)'s straw poll to the laboratory. Much more generally, Gerardi and Yariv (2007) study sequential equilibria that are implementable by a centralized mediator, which is outcome equivalent to studying all cheap-talk extensions of the voting game and hence covers arbitrary communication protocols. In an application, they show that one round of public messages prior to the vote admits the same set of (sequential) equilibria for a whole set of voting rules. Intuitively, the information aggregation that the vote has to achieve in the standard model is shifted up the game tree and is now obtained in the communication stage already. This effect finds strong empirical support in a set of experiments reported in Goeree and Yariv (2011). ${ }^{11}$

By contrast, introducing a public signal on the state of the world prior to the vote changes the picture dramatically. Kawamura and Vlaseros (2017), Liu (2019) and Jeong (2019) find that the presence of a public signal generates a new class of equilibria in which voters discard their private information in favor of the public signal and information aggregation is inefficient, even if voters condition their strategy on pivotality. ${ }^{12}$

We consider a third way of correlating voters' information in the standard model of common-interest voting: private communication between voters in the form of vote recommendations. To our knowledge, private communication before voting in a common-interest setting has so far been modeled either implicitly by studying (with a mediator) all cheap-talk extensions of the voting game (Gerardi and Yariv, 2007; Iaryczower et al., 2018) or as repeated exchange among naïve voters who do not condition on pivotality (Golub and Jackson, 2012). ${ }^{13}$ We show that the way in which private communication affects information aggregation is different from the effects of public communication: Although efficient equilibria always exist (in particular, the LTED equilibrium), there are also equilibria (in particular, the sincere equilibrium) in which information is inefficiently aggregated, given that the network is not strongly balanced. The latter equilibria and their corresponding "sincere" strategies are more frequently played in the laboratory than the former. Hence, private communication indeed undermines informational efficiency if some voters are too powerful communicators. After generalizing the model to admit all possible network structures, our model incorporates vote recommendations that are both private and public, and hence builds a bridge between private communication and public communication in the form of vote recommendations. $^{14}$

Organization of the paper. In the next section, we introduce a simple model of vote recommendations, restricting our analysis to a specific subset of communication networks and to homogeneous signal qualities. We characterize conditions on the network structure under which the two focal strategy profiles, i.e., sincere behavior and LTED, are equilibria. In section 3 , we present the design of the laboratory experiment and in section 4 , we report the experimental results. In section 5 , we conclude. In Appendix A.2, we analyze the general model with arbitrary networks and heterogeneous signal precisions and prove our theoretical results. In the supplementary online material (henceforth: SOM), we provide supplementary mathematical results (in SOM B) and supplementary experimental material including the instructions (in SOM C).

\section{A simple model of vote recommendations}

\subsection{Set-up}

Nature draws one state of the world, $\omega$, which has two possible realizations, $A$ and $B$, that occur with equal probability and are not directly observable. There is a finite set of agents partitioned into a group of experts $M$ and a group of non-experts $N$. Respective group sizes are $m=|M|$ and $n=|N|$. Experts $j \in M$ receive a private independent signal $s_{j} \in\left\{A^{*}, B^{*}\right\}$ about the true state of the world. The signal is imperfectly informative with quality $p_{j}=\operatorname{Pr}\left\{s_{j}=A^{*} \mid \omega=A\right\}=$

\footnotetext{
10 In a recent theory paper, Battaglini (2017) allows for communication between citizens in separate audiences so that information becomes correlated among the citizens in one audience. However, in his model, citizens cannot vote on policies directly but coordinate on public protest instead, potentially signing a petition against the policy maker's default policy. Battaglini shows that communication in social media can improve information aggregation and transmission via public protests.

11 By implementing free text communication in the laboratory, Goeree and Yariv (2011) can study which kind of communication emerges. They find that participants choose public communication, first to reveal their private information (deliberation), then to prepare decision making. This fosters efficiency, as predicted by the theory on deliberation with public communication above. Hence, the study of private information applies to settings where there are exogenous restrictions on the communication channels (such as in large elections).

12 Somewhat relatedly, the literature on hidden profiles reports that in group discussions prior to group decisions, information shared with other group members gets too much weight compared to unique private information. See, e.g., the meta-study by Lu et al. (2012).

13 When admitting arbitrary communication protocols, Iaryczower et al. (2018) demonstrate positive and negative effects of pre-vote communication on information aggregation in a jury. For a society of naïve learners, it is known that information aggregation is inefficient in the long term when some agents are overly powerful (Golub and Jackson, 2010). In their application of the homophily theory to voting, Golub and Jackson (2012) assume that every voter has the same expected number of links and is hence equally influential. Then information aggregation is efficient in the limit, but not necessarily in the intermediate term: They show that inefficiency can arise, when there are multiple groups with more links within group than across groups (i.e., homophily) and when the signals are not distributed equally across groups.

14 There are other forms of communication that we do not address in this paper. They include multi-round communication, discussions about signal quality or reasoning, discussions about decision making, differentiated messages for different recipients, etc.
} 
$\operatorname{Pr}\left\{s_{j}=B^{*} \mid \omega=B\right\} \in\left(\frac{1}{2}, 1\right)$. We preliminarily assume that $p_{j}=p \forall j \in M$ and that non-experts $i \in N$ do not receive a signal, but can potentially receive a message from an expert. A graph $g$ represents the communication structure between non-experts and experts. We preliminarily assume that $g$ is bipartite, consisting of links $(i, j) \subseteq N \times M$ only. ${ }^{15}$ Degree $d_{i}$ is the number of links of agent $i$. An expert $j$ with $d_{j} \geq 1$ is called sender and all non-experts linked to $j$ are called the "audience of $j$." Our final preliminary assumption is that different audiences do not overlap, i.e., the degree of each nonexpert is at most one, such that no agent can access more than one piece of information. ${ }^{16}$ We will drop our preliminary assumptions in section 2.4 and Appendix A.2.

After receiving the signal, each sender may send message "A" or message "B" or an empty message $\emptyset$ to her audience. Then, all agents participate in a majority vote the outcome of which determines which of two alternative policies, $P_{A}$ or $P_{B}$, shall be implemented. Voters simultaneously vote for one of the two policies or abstain. If one policy obtains a simple majority, i.e., a plurality, of votes, it is implemented; otherwise, the policy to be chosen is randomly drawn with equal probability from the two alternatives.

All experts and non-experts are assumed to be unbiased in the sense that they want the policy to match the state of the world. More precisely, their utility is represented by $u\left(P_{A} \mid A\right)=u\left(P_{B} \mid B\right)=1$ and $u\left(P_{B} \mid A\right)=u\left(P_{A} \mid B\right)=0 .{ }^{17,18}$

The sequence of actions is as follows. First, nature draws the state of the world and the signals of the experts. Second, each sender decides which message to communicate to her audience, if any. Third, all agents vote or abstain and the outcome is determined by the simple-majority rule. The full description of the game including the network structure is common knowledge. ${ }^{19}$

Strategies are defined as follows: A communication and voting strategy $\sigma_{j}$ of a sender $j \in M$ defines which message to send and whether and how to vote for each signal received, i.e., $\sigma_{j}:\left\{A^{*}, B^{*}\right\} \rightarrow\{A, B, \emptyset\} \times\{A, B, \emptyset\}$ if $d_{j} \geq 1$ and $\sigma_{j}:\left\{A^{*}, B^{*}\right\} \rightarrow\{A, B, \varnothing\}$ if $d_{j}=0$. We can abstract from the timing of these two actions (communication and voting) here. A voting strategy of a non-expert $i \in N$ with a link is a mapping from the set of messages into the voting action $\sigma_{i}:\{A, B, \varnothing\} \rightarrow\{A, B, \varnothing\}$, and a voting strategy of an agent $i \in N$ without a link is simply a voting action $\sigma_{i} \in\{A, B, \emptyset\}$. A strategy profile $\sigma$ consists of all experts' and all non-experts' strategies.

We analyze this model using the concept of perfect Bayesian equilibrium, i.e., agents use sequentially rational strategies, given their beliefs, and beliefs are updated according to Bayes' rule whenever possible. We focus on two strategy profiles, one with information transmission ("sincere") and one without information transmission ("let the experts decide", in short: LTED). These are the two strategy profiles predominantly discussed in the literature on voting. Still, communication generally gives rise to a multiplicity of equilibria, and focality of strategies is ultimately an empirical question. We address this question in our laboratory experiment. It turns out that exactly the two strategy profiles that we focus on are the relevant ones in the laboratory.

Note that if all non-experts in a given audience choose not to condition their voting action on the message received, then the outcome of the game is as if communication was not possible at all ("babbling equilibrium"). Similarly, if all nonexperts in a given audience vote $B$ if the message is $A$ and vote $A$ if the message is $B$, then the outcome of the game is as if their sender had chosen another communication strategy, where messages $A$ and $B$ are permuted ("mirror equilibria"). We will not differentiate between mirror equilibria, i.e., differentiate equilibria on the basis of the syntax of information transmission. Instead, we will identify equilibria via the semantics of information transmission, i.e., on the basis of the meanings that messages acquire in equilibrium. ${ }^{20}$

A desirable property of an equilibrium is informational efficiency which is defined as follows.

Definition 2.1. A strategy profile $\sigma$ is efficient if it maximizes the ex ante probability of the implemented policy matching the true state of the world.

Observe that an efficient strategy profile $\sigma$ maximizes the sum of ex ante expected utilities of all experts and nonexperts since they are unbiased. Given efficient strategy profiles, the probability of matching the true state is maximized but not equal to one because it might always happen by chance that many experts receive the wrong signal. Letting the number of experts grow, this probability approaches one as in Condorcet's Jury Theorem. Observe also that in this simple setting an efficient strategy profile is characterized by always implementing the policy indicated by the signal that has been received by most experts, which we call the majority signal. For convenience, we let the number of experts $m$ be odd such that there is always a unique majority signal indicating the policy that should be implemented. ${ }^{21}$

\footnotetext{
15 The network is undirected since it represents the communication channels that can be potentially used.

16 This assumption assures that information aggregation can only take place in the voting stage but not in the communication stage and hence is the natural counterpart to public communication and deliberation.

17 Here, we follow the convention to define cardinal utility levels, although this assumption is not necessary.

18 An extended model with heterogeneous preferences, in particular with biased agents who always favor one of the two alternatives, is studied in SOM C.3

19 Knowing the network structure prevents potential inefficiencies due to imperfect information about the network structure.

20 This is standard in the cheap-talk literature starting with Crawford and Sobel (1982).

21 Admitting an even number of experts would not change the results qualitatively, but it would make the analysis cumbersome because more cases would have to be distinguished.
} 
While the definition of informational efficiency above is binary, strategy profiles can also be ranked according to their informational efficiency by comparing their corresponding ex ante probabilities of matching the true state.

Hereafter, we will slightly misuse notation by using " $A$ " and " $B$ " to denote the corresponding state of the world, message content, and policy, whenever the context prevents confusion.

\section{2. "Let the experts decide"}

One important feature of this simple model is that informational efficiency can always be obtained in equilibrium, regardless of the network structure. Consider for instance the strategy profile $\sigma^{*}$ in which all experts vote in line with their signal and all non-experts abstain. Under the simple-majority rule this LTED strategy profile $\sigma^{*}$ is efficient since for any draw of nature the signal received by a majority of experts is implemented. Moreover, because preferences are homogeneous, efficient strategy profiles do not only maximize the sum of utilities, but also each individual agent's utility. Thus, there is no room for improvement (as already argued in McLennan, 1998).

Proposition 2.1. There exist efficient equilibria for any network structure. For instance, the LTED strategy profile $\sigma^{*}$ is efficient and an equilibrium for any network structure.

Importantly, while every efficient strategy profile constitutes an equilibrium, the reverse does not hold true. On the contrary, there are (trivial and non-trivial) inefficient equilibria of the game. One non-trivial inefficient equilibrium will be discussed as Example 3 below.

Among the efficient equilibria, we consider the LTED equilibrium $\sigma^{*}$ focal for two reasons. First, it is simple: All experts use the same type of strategy and all non-experts use the same type of strategy. Second, it is intuitive to abstain as a non-expert and to vote in line with one's signal as an expert, as already argued by, e.g., Feddersen and Pesendorfer (1996) and experimentally shown by Morton and Tyran (2011). However, since it is also intuitive for experts to send informative messages and for receivers to vote according to their messages, it may nonetheless be difficult to coordinate on $\sigma^{*}$. In particular, consider the strategy profile $\hat{\sigma}$ in which experts communicate and vote for the policy indicated by their signal and non-experts vote in line with their message and abstain if they did not receive any information. This strategy profile $\hat{\sigma}$ is sincere in the sense that each agent communicates and votes for the alternative that she considers as most likely given her private information. ${ }^{22}$ We now proceed by investigating the sincere strategy profile.

\subsection{Sincere voting}

Balanced networks. To characterize under which conditions on the network structure the sincere strategy profile $\hat{\sigma}$ is an equilibrium, we define two intimately related balancedness requirements. Both the content and the purpose of the following definition will be explained with the help of simple examples below.

Definition 2.2 (Balancedness). We define strong and weak balancedness.

(a) Let $M^{\prime} \subset M$ denote the set of the $m^{\prime}=\frac{m+1}{2}$ experts with the lowest degree. ${ }^{23}$ A network is called "strongly balanced" if this set is involved in at least half of all links, i.e. $\sum_{j \in M^{\prime}} d_{j} \geq \sum_{k \in M \backslash M^{\prime}} d_{k}$.

(b) For an expert $j \in M$, let $\mathcal{M}_{j}$ be the set of expert sets $M^{\prime \prime} \subseteq M$ that contain expert $j$ and form a slight majority when adding their audiences of non-experts, i.e. $\sum_{k \in M^{\prime \prime}}\left(d_{k}+1\right)-\sum_{l \in M \backslash M^{\prime \prime}}\left(d_{l}+1\right) \in\{0,1,2\}$. A network is called "weakly balanced" if for every expert $j \in M$, non-emptiness of this set, i.e. $\mathcal{M}_{j} \neq \emptyset$, implies that there is at least one element consisting of a weak majority of experts, i.e. $\exists M^{\prime \prime} \in \mathcal{M}_{j}$ such that $m^{\prime \prime} \geq \frac{m+1}{2}$.

To illustrate strong and weak balancedness, we use the following two examples.

Example 1. Let $n=4, m=5$, and the degree distribution of experts $\left(d_{1}, d_{2}, d_{3}, d_{4}, d_{5}\right)=(1,1,1,1,0)$ as illustrated in the left panel of Fig. 1. This network is strongly balanced since $d_{3}+d_{4}+d_{5} \geq d_{1}+d_{2}$; and it is weakly balanced since every slight majority of voters in which a given expert $j \in\left\{j_{1}, j_{2}, j_{3}, j_{4}, j_{5}\right\}$ partakes comprises a weak majority of experts, too.

Example 2. Let $n=4, m=5$, and the degree distribution of experts $\left(d_{1}, d_{2}, d_{3}, d_{4}, d_{5}\right)=(4,0,0,0,0)$ as illustrated in the right panel of Fig. 1. This network violates weak balancedness. Indeed, $\mathcal{M}_{1}=\left\{\left\{j_{1}\right\}\right\}$ such that there is no $M^{\prime \prime} \in \mathcal{M}_{1}$ with $m^{\prime \prime} \geq \frac{m+1}{2}=3$. Put differently, expert 1 can partake in a slight majority of voters that contains only a minority of experts (himself)

\footnotetext{
22 The LTED strategy profile $\sigma^{*}$, in contrast, is not "fully sincere" for the following reason. The aspect that information is not transmitted either means that senders do not communicate their signal or that receivers do not follow their message.

23 If there are several of these sets, we can choose any one. If $m$ was even, we would require $m^{\prime}=\frac{m+2}{2}$ in this definition.
} 

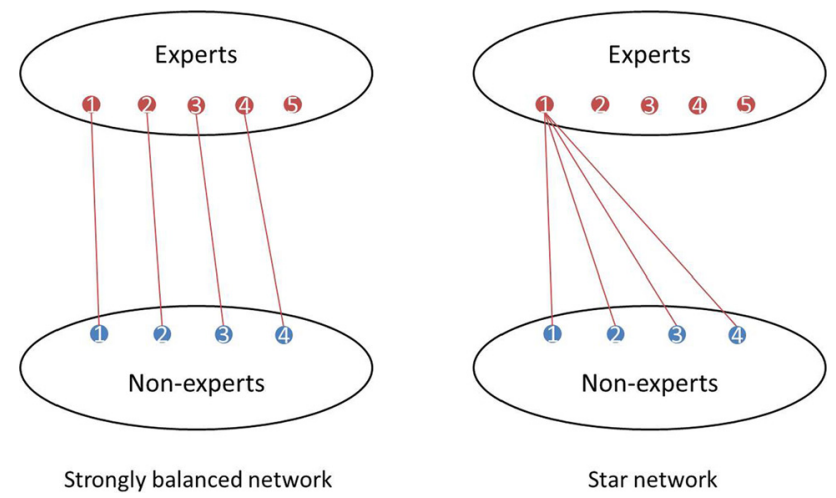

Fig. 1. Left: Example 1, which is a network satisfying strong balancedness. Right: Example 2, the star network, which is an unbalanced network.

In general, strong balancedness requires that even the majority of experts with the smallest degrees, which is called $M^{\prime}$ in the definition, is involved in at least half of all links. This simply means that every majority of experts is involved in at least half of all links, which requires a rather even degree distribution. ${ }^{24}$ Weak balancedness restricts a related requirement to certain sets of experts $\mathcal{M}_{j}$. Indeed, strong balancedness always implies weak balancedness (since it implies for all $j$ and all $M^{\prime \prime} \in \mathcal{M}_{j}$ that $m^{\prime \prime} \geq \frac{m+1}{2}$ ). Networks violating weak balancedness also violate strong balancedness and will be called unbalanced hereafter.

The two properties capture some kind of balance between a group's expertise (which depends on the number of signals) and its power (which depends on the size of the audiences). For instance, in Example 1, experts are equally powerful, whereas in Example 2, expert 1 is overly powerful, compared to the other experts. ${ }^{25}$

Proposition 2.2. The sincere strategy profile $\hat{\sigma}$ is efficient if and only if the network is strongly balanced. The sincere strategy profile $\hat{\sigma}$ is an equilibrium if (a) the network is strongly balanced, and only if $(b)$ the network is weakly balanced.

Applied to our two examples, Proposition 2.2 implies that the sincere strategy profile $\hat{\sigma}$ is efficient and an equilibrium in Example 1 but neither efficient nor an equilibrium in Example 2. The intuition of Proposition 2.2 can be illustrated with these two examples. ${ }^{26}$

Consider first strong balancedness in Example 1. Observe that under the sincere strategy profile $\hat{\sigma}$ any three experts who vote and communicate the same alternative determine the final outcome. Thus, for any draw of nature the policy indicated by the majority signal is implemented, which means that information is aggregated efficiently and hence $\hat{\sigma}$ is an equilibrium. Likewise, in any strongly balanced network the majority signal receives a majority of votes. The reason is that the set of experts who have received this signal has a majority of votes when considering their own votes and the votes of their audiences.

Consider now weak balancedness, which is violated in Example 2. To see why $\hat{\sigma}$ is inefficient in Example 2, consider a draw of nature by which the most powerful expert, i.e., the expert $j_{1}$ with the highest degree, receives the minority signal. Assume now, for the sake of argument, that the sincere strategy profile $\hat{\sigma}$ is played. In this case the minority signal determines which policy is implemented; information is hence aggregated inefficiently. To see why $\hat{\sigma}$ is not an equilibrium, consider the following two deviation incentives. First, the most powerful expert would want to deviate to not communicating, but still voting for, the policy indicated by her signal. This would lead to an efficient strategy profile that is outcome-equivalent to LTED since the non-experts then abstain. Second, the non-experts, too, can improve by deviating. In particular, consider a non-expert receiving message $A$. His posterior belief that $A$ is true is $p_{i}(A \mid A)=p>\frac{1}{2}$. However, his posterior belief that $A$ is true, given that he is pivotal, is $p_{i}(A \mid A, p i v)<\frac{1}{2}$ because in this simple example pivotality only occurs when all other experts have received signal $B^{*}$. Thus, abstention or voting the opposite of the message is a strict improvement for any non-expert.

Example 2 provides a simple illustration of the swing voter's curse. The argument, however, is much more general. Assume that all agents play according to the sincere strategy profile $\hat{\sigma}$ and consider the receivers who belong to a large audience. These receivers know that their sender is very powerful. Hence, if they are pivotal in the vote, this implies that a considerable number among the other experts must have got a signal that contradicts the message they received. Thus, if

\footnotetext{
24 This requirement follows from the assumption in the simple model that the signal quality of the experts is homogeneous. In the general model with heterogeneous signal quality, networks with unequal degree distributions can also be strongly balanced as it will become clear in Appendix A.2.

25 A formal definition of power is given in SOM B.4. It relies on the cooperative framework of simple games, in which individual power is measured by the Shapley-Shubik index or the Banzhaf index, which both count the number of "swings" a voter has (cf., e.g., Roth, 1988).

26 Note that Proposition 2.2 provides one sufficient and one necessary condition for the sincere strategy profile $\hat{\sigma}$ to be an equilibrium, but no condition that is both sufficient and necessary. For such a condition see Proposition B.1 in SOM B.1.
} 


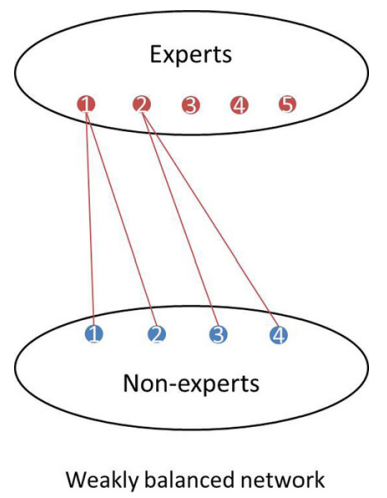

Fig. 2. Example 3, a network in which the sincere strategy profile $\hat{\sigma}$ is both inefficient and an equilibrium.

following the message has any effect on the outcome, it has most likely a detrimental effect. If a receiver realizes that he is "cursed" in this sense, he wants to deviate from the sincere strategy and prefers to abstain or to vote the opposite.

Inefficient equilibria. For networks that satisfy the necessary condition (weak balancedness), but violate the sufficient condition (strong balancedness) the sincere strategy profile $\hat{\sigma}$ is inefficient but potentially still an equilibrium. More generally, the question arises whether there are equilibria with information transmission prior to the vote that are inefficient.

Proposition 2.3. There are networks in which the sincere strategy profile $\hat{\sigma}$ is both an equilibrium and exhibits informational inefficiency. This inefficiency does not necessarily vanish when the number of experts and the number of non-experts grow large.

One example demonstrating the above proposition is given below.

Example 3 (weakly balanced). Let $n=4, m=5$, and the degree distribution of experts $\left(d_{1}, \ldots, d_{5}\right)=(2,2,0,0,0)$ as illustrated in Fig. 2. In this network the sincere strategy profile $\hat{\sigma}$ is inefficient because the network violates strong balancedness. However, we can show that the sincere strategy profile $\hat{\sigma}$ is an equilibrium in this network. Moreover, this inefficiency need not vanish for large numbers of experts and non-experts. Let the network grow infinitely in discrete steps $t=1,2,3, \ldots$ by adding two non-experts to each audience and two experts without an audience in each step. ${ }^{27}$ For any $t \geq 1$ the sincere strategy profile $\hat{\sigma}$ is an equilibrium in this example and the inefficiency does not converge to zero for $t$ going to infinity (see proof of Proposition A.3 in Appendix A.2).

Overall, we can conclude that communication need not, but can impair information aggregation in equilibrium, depending on the balancedness of the network structure. In strongly balanced networks (such as in Example 1), $\hat{\sigma}$ is both efficient and an equilibrium. In weakly balanced networks that are no longer strongly balanced (such as in Example 3 ), $\hat{\sigma}$ can still be an equilibrium, but is always informationally inefficient. Finally, in unbalanced networks (such as in the star network, Example 2) neither property holds. There the swing voter's curse occurs such that non-experts can profitably deviate from $\hat{\sigma}$ by not following their message.

\subsection{Robustness in the general model and in the limit}

In Appendix A.2, we generalize the above model in two ways. First, we assume that every agent $i$ receives a signal with idiosyncratic signal quality $p_{i} \in\left[\frac{1}{2}, 1\right)$. Second, we admit arbitrary network structures. Hence, agents can receive multiple messages. We show in the appendix that our theoretical results (Propositions 2.1-2.3) are robust in the following sense.

In all networks, there exists a LTED equilibrium (with "experts" re-defined as all agents with a signal quality above a cut-off) ${ }^{28}$ We provide a sufficient and necessary condition under which this equilibrium is efficient and show that, even if this condition is not fulfilled, the former equilibrium is asymptotically efficient if the size of the electorate goes to infinity (Proposition A.1). We generalize the definition of the sincere strategy profile (voters recommend the policy that corresponds to their own signal to their neighborhood, update their beliefs according to Bayes' rule, and vote for the policy that is more likely to be optimal, given their updated beliefs). We also generalize the definitions of strong and weak balancedness and re-state our result that the sincere strategy profile is an equilibrium if the network is strongly balanced and only if it is weakly balanced (Proposition A.2). We then demonstrate our main result which states that the inefficiency of (some) sincere-strategy equilibria subsists in the limit (Proposition A.3).

\footnotetext{
27 The $t$-th network then has two experts of degree $2 t$ and $2 t+1$ experts of degree zero.

28 The choice of the cut-off, which defines the experts, is arbitrary, as long as it is strictly above 0.5 and the induced number of experts is odd.
} 


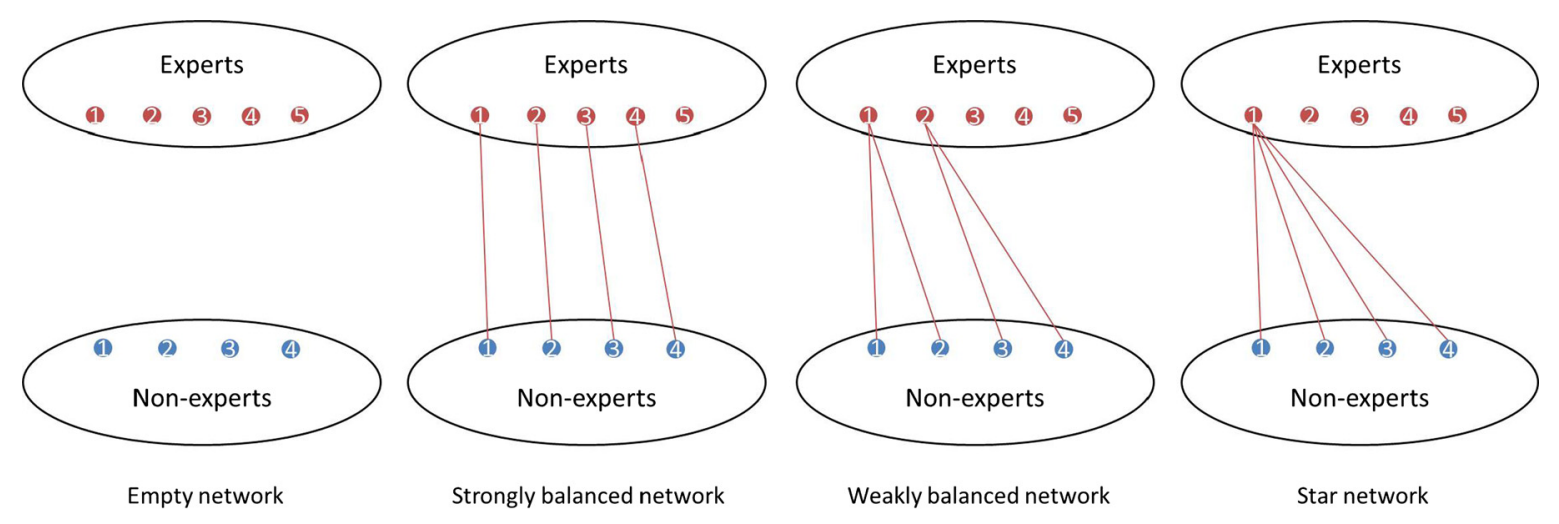

Fig. 3. The four treatments. The empty network is the baseline treatment. The strongly balanced network is Example 1. The weakly balanced network is Example 3. The star network is Example 2. The sincere strategy profile $\hat{\sigma}$ is an equilibrium in the strongly and the weakly balanced network; it is efficient in the strongly balanced network. LTED $\sigma^{*}$ is an efficient equilibrium in any network.

\section{Experimental design}

Motivation of the experiment. Whether real people, both individually and collectively, account for the swing voter's curse in unbalanced networks is an empirical question. Therefore, we bring the theory to the laboratory and find out how experimental subjects play the game in various networks that differ in their balancedness. The other, equally important, purpose of the laboratory experiment is to test our focality assumption and to empirically study (network-dependent) equilibrium selection. In particular, in the case of weakly balanced networks that are not strongly balanced the quality of information aggregation depends on whether the agents manage to coordinate on the efficient LTED equilibrium or whether they coordinate on the inefficient sincere equilibrium, or on other potential equilibria.

To theoretically prepare experimental equilibrium selection, we address the question of additional, non-focal equilibria. We extend the equilibrium analysis of our Examples 1, 2, and 3, which is particularly useful since these examples are also implemented in our experiment. In SOM B.2, we give a full characterization of all equilibria conforming to four selection criteria (Purity, Symmetry, Monotonicity, and Neutrality). It shows that one more strategy than considered so far contributes to equilibrium formation, namely a delegation strategy according to which experts with an audience delegate their vote to their audience by revealing their signal and abstaining themselves. Moreover, there are equilibria in which experts who are never pivotal abstain from voting without delegating their vote. However, there are no additional strategies that arise as composites of equilibria in these examples. All equilibria conforming to our selection criteria are composites of the LTED strategy profile, the sincere voting profile, and the delegation or abstention strategies of experts.

Experimental implementation. We conducted two experiments. In the main text, we report the results of the one that directly tests the model above. ${ }^{29}$ Our experimental design implements the empty network, in which communication is precluded, and the three examples - Example 1,2, and 3 - analyzed above. These four different communication networks are depicted in Fig. 3. The empty network serves as a benchmark, since the sincere strategy profile $\hat{\sigma}$ is impossible to play when there are no communication channels. Hence, the LTED equilibrium is the only focal equilibrium in the empty network. The other three networks differ in the way described in section 2.3. In particular, the sincere strategy profile is an efficient equilibrium in the strongly balanced network; it is an inefficient equilibrium in the weakly balanced network; and it is neither an equilibrium nor efficient in the star network.

Each of these networks corresponds to one experimental treatment; and treatments are varied within subjects (i.e., all participants in a given session play the communication and voting game in all four networks) in random order. Voter groups - i.e., subject groups interacting in one network - consist of five experts and four non-experts. Apart from the baseline treatment, the empty network, the density of the networks is held constant while the equality of the degree distribution is decreasing.

The experiment was conducted in the WISO-lab of the University of Hamburg, using the software z-Tree. We ran seven sessions with $3 * 9=27$ participants in each session. All subjects in a session played the game described above in all four networks over 40 rounds in total. Each of the four treatments occurred in two blocks of five subsequent rounds. Blocks were ordered randomly. For the recruitment of a total of 189 subjects, we used the software tool hroot (Bock et al., 2014). Virtually all subjects were undergraduate or master students at the University of Hamburg from a variety

\footnotetext{
29 The second experiment, Study II, tests an extended model with fully analogous propositions. It shows that our findings, as reported below, are fully robust. Each result - with one notable exception - is matched by a corresponding result in Study II. If anything effects are even stronger and significant on a higher level in Study II. The exception concerns the communication behavior of the sender in the star network, which in Study II is truthful independent of the treatment. Study II can be found in SOM C.3 referring to an earlier working paper version that is permanently available online (Buechel and Mechtenberg, 2017).
} 
Table 1

Equilibrium Selection.

\begin{tabular}{|c|c|c|c|c|c|}
\hline & Equilibria & Equ. Play & Sincere $\hat{\sigma}$ & LTED $\sigma^{*}$ & All others \\
\hline empty $(N=210)$ & $\sigma^{*}$ & 23 & - & 23 & 0 \\
\hline strongly balanced $(N=210)$ & $\sigma^{*}, \hat{\sigma}$, and 17 others & 44 & 21 & 0 & $23^{a}$ \\
\hline weakly balanced $(N=210)$ & $\sigma^{*}, \hat{\sigma}$, and 7 others & 18 & 16 & 2 & 0 \\
\hline $\operatorname{star}(N=210)$ & $\sigma^{*}$, and 5 others & 5 & $(5)^{b}$ & 5 & 0 \\
\hline Total $(N=840)$ & & 90 & $37(42)$ & 30 & 23 \\
\hline
\end{tabular}

Notes: Frequency of equilibria. The column 'Equilibria' lists the strategy profiles that comprise an equilibrium and that satisfy our equilibrium selection criteria (Purity, Symmetry, Monotonicity, and Neutrality). The other columns report the empirical frequency of certain equilibria, i.e., how often all actions in a group are consistent with an equilibrium strategy profile.

$a$ : The 23 times that other equilibria than LTED $\sigma^{*}$ and sincere $\hat{\sigma}$ were played, it was each time the same equilibrium. Its strategy profile differs from the sincere strategy profile in that one of the four non-experts abstains. In SOM B.2 this equilibrium is denoted by $v_{1}, r=3$ and illustrated in Fig. B.1, Panel (a). $b$ : In the star network, the strategy profile sincere $\hat{\sigma}$ is not an equilibrium, but played 5 times.

of fields. None of them had previously participated in a related experiment. At the beginning of each session, subjects randomly received the role of an expert or the role of a non-expert. These roles were fixed throughout the experiment. ${ }^{30}$ In each round, subjects were randomly matched into groups of nine (five experts and four non-experts). At the end of each round, the participants learned the chosen policy, the true state, and the voter turnout in their group. They did not learn individual votes, vote shares per role, or any behavior from other groups. Groups were newly formed each round by random re-matching. Instructions that described the experimental session in detail were handed out at the beginning of each session and were followed by a short quiz that tested the subjects' understanding of the game. ${ }^{31}$ Moreover, there were four practicing rounds, one for each treatment, that were not payout-relevant. During the entire session, each subject always knew his own network position and the structure of the network. The quality of the signal that the experts received was $p=0.6$. At the end of each session, three rounds were randomly drawn and payed out in cash and in private. Subjects earned 5 Euros for a correct group choice and 0 Euros for an incorrect group choice. Hence, a given subject could earn either 0 , or 5 , or 10 , or 15 Euros from group play, depending on how many of the three randomly drawn payoff-relevant rounds were associated with a correct group choice for this particular subject. In addition, each subject received a show-up fee of 5 Euros. On average, sessions lasted for 1.5 hours and subjects earned EUR $14.3 .{ }^{32}$

\section{Experimental results}

Table 2 in Appendix A.1 gives a summary of the number of observations. On the group level we have 840 observations. On the individual level we have 7,560 observations with 40 decisions per subject. In total, 189 subjects participated in the experiment.

After analyzing equilibrium selection in section 4.1, we will turn to individual behavior in section 4.2. We address efficiency in section 4.3. The experimental results are illustrated in the main text by figures. Most tables are relegated to Appendix A.1.

\subsection{Equilibrium selection}

Focality. Since our setting gives rise to a multiplicity of equilibria, the first question is which equilibria are played. We check how frequently actual behavior in a group is consistent with one of the existing equilibrium strategy profiles (Table 1). It turns out that the empirical frequency of most equilibria is $0.0 \%$ : Out of the 840 observations on the group level, respectively out of the 210 group observations per treatment, they are not even played once. There are two equilibria which are frequently played, however: the sincere $\hat{\sigma}$ and the LTED $\sigma^{*} \cdot{ }^{33}$ Besides these two focal equilibria there is only one more equilibrium that is actually ever played. This is an equilibrium in the strongly balanced network, which is highly similar to sincere behavior, but with the difference that one of the four non-experts abstains. ${ }^{34}$ Hence, by focusing on the sincere strategy profile and the LTED strategy profile, we do not miss other relevant equilibria.

Moreover, we counted to which extent the two focal strategy profiles can predict the actual group decision given the actual distribution of signals. In every treatment more than $80 \%$ of the actual outcomes are predicted by at least one of the

\footnotetext{
30 The network position varied within roles; e.g., the expert at the center of the star network was randomly drawn from among all experts in the group at the beginning of each round in which the star was played.

31 The instructions and the quiz can be found in SOM C.2.

32 The norm in the WISO-lab at the University of Hamburg was EUR 10 per hour.

33 Sometimes there is more than one equilibrium strategy profile that induces LTED.

34 In SOM B.2 this equilibrium is denoted by $v_{1}, r=3$ and illustrated in Fig. B.1, Panel (a).
} 


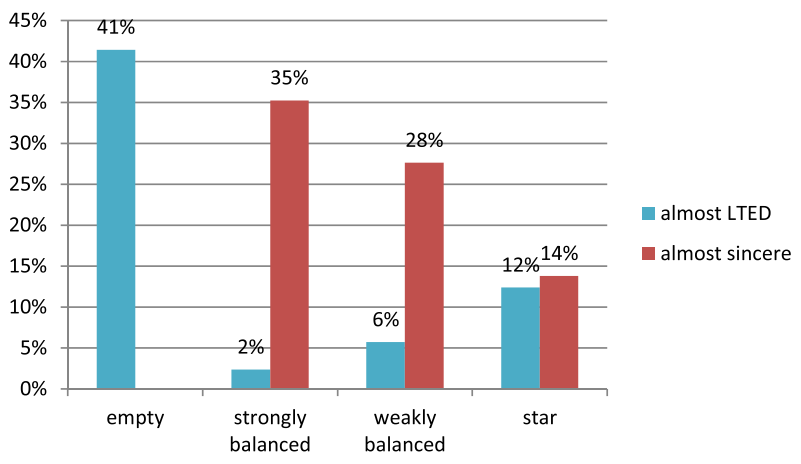

Fig. 4. Frequency of behavior consistent with strategy profiles $\sigma^{*}$ and $\hat{\sigma}$ by treatment. A strategy profile is "almost" played if at most one agent has chosen a different strategy. All differences - apart from the comparison between the strongly and weakly balanced networks - are significant on the $p<0.05$ level (Tables 4 and 5).

two focal strategy profiles (Table 3, last column). This is another indication that the focus on the two focal equilibria is well justified by the data. ${ }^{35}$

Treatment effects on equilibrium selection. The second question is how the network structure affects which strategy profile is selected. Fig. 4 (and Tables 1 and 3) show the results. Since many groups' strategy profiles are not consistent with any equilibrium, we additionally consider playing almost a strategy profile, when at most one of the nine subjects in a group has chosen a different strategy. ${ }^{36}$ This gives a more exhaustive view on group behavior.

In the empty network, in which $\hat{\sigma}$ cannot be played, we find the highest level of coordination on $\sigma^{*}$. In the strongly balanced network there are 19 different equilibria, including the two focal ones, which are both efficient in this network. Behavior is consistent with only two equilibria: the sincere $\hat{\sigma}$ and the other equilibrium that is highly similar to sincere. Hence, the (almost) sincere strategy profile $\hat{\sigma}$ is predominant, while LTED $\sigma^{*}$ is rarely ever played. In the weakly balanced network, there are 9 different equilibria, including the two focal ones. LTED $\sigma^{*}$, which is efficient, is rarely played. The sincere equilibrium $\hat{\sigma}$, which is inefficient, is more frequently selected. Hence, in both balanced networks equilibrium selection is in favor of the sincere strategy profile. In the star network, LTED $\sigma^{*}$ is the only equilibrium that is ever played. However, we note that the sincere strategy profile $\hat{\sigma}$, which is not an equilibrium and inefficient in this network, is still played as frequently as LTED.

Comparing across treatments, network balancedness leads to a higher frequency with which groups coordinate (almost) on the sincere strategy profile $\hat{\sigma}$ and to a lower frequency with which groups coordinate (almost) on the LTED strategy profile $\sigma^{*}$. Fisher exact tests reveal that these differences are significant on the $p<0.05$ level when taking the "almost" definition of the strategy profiles and most of them are also significant when taking the strict definition (Tables 4 and 5). The following result summarizes the findings.

Result 1. In the (strongly and weakly) balanced networks, groups coordinate more often on the sincere strategy profile $\hat{\sigma}$ than on LTED equilibrium $\sigma^{*}$. In the star network groups coordinate less often on $\hat{\sigma}$ and more often on $\sigma^{*}$ than in the balanced networks. Coordination on $\sigma^{*}$ is highest in the empty network.

The selection of the sincere strategy profile in the weakly balanced network means that the inefficient equilibrium is selected. The comparison between the balanced networks and the star network is in line with the theoretical prediction (since $\hat{\sigma}$ is not an equilibrium in the unbalanced star network). ${ }^{37}$

In sum, we find that our focality assumption on equilibrium selection is well supported by our experimental findings. Moreover, we find that equilibrium selection is more in favor of the sincere strategy profile. Given this finding, the resulting comparative-static predictions of the theory are supported as well. ${ }^{38}$

\footnotetext{
35 Finally, there could be additional equilibria involving mixed strategies. In our analysis of the experimental data we have not found any indication for the relevance of mixed strategies. When investigating individual behavior of the same subjects in different instances (such as in Fig. 7), it turns out that many agents consistently choose the same action when they are in the same treatment.

36 Our within-subjects design with stranger matching makes it very demanding for subjects to coordinate.

37 When exploring learning effects by restricting attention to the second half of rounds, i.e., round 21 to 40 , Result 1 still holds. Moreover, in comparison with the first half of rounds, i.e., round 1 to 20 , there is higher coordination on $\sigma^{*}$ in the star network and lower coordination on $\hat{\sigma}$ in the balanced networks in the second half. Both changes could be interpreted as learning effects since agents play more frequently the efficient equilibrium and less frequently the strategy profile that is inefficient in the weakly balanced network.

38 These findings are particularly strong since our experimental design is (intentionally) biased against them: the within-subjects design with stranger matching makes it difficult for subjects to coordinate at all, let alone conditional on treatment, and still we find significant treatment-conditional equilibrium selection.
} 


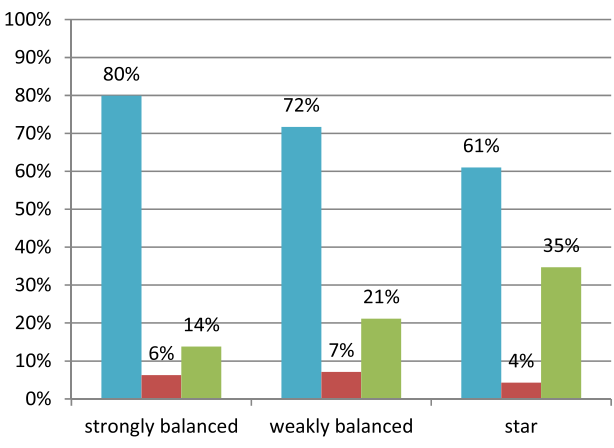

send signal

w send opposite

and empty

Fig. 5. Frequency of experts' communication behavior by treatment. 'Send signal' means to send message $A(B)$ when the signal received is $A^{*}\left(B^{*}\right)$. 'Send opposite' means to send message $A(B)$ when the signal received is $B^{*}\left(A^{*}\right)$. Differences are significant on the $p<0.05$ level (cf. Table 7).

\subsection{Individual behavior across networks}

Experts. Around $80 \%$ of the time experts vote and communicate in accordance with their signal, i.e., they play the sincere strategy $\hat{\sigma}_{j}$ (Table 6). While experts vote in line with their signal in a large majority of instances, there are some deviations from the sincere strategy profile on the communication stage, as can be seen from Fig. $5 .{ }^{39}$ Recall that there is an incentive to deviate in the star network since it is unbalanced. Indeed, information transmission is lowest in the star network, where only $61 \%$ of the senders communicate their signal, whereas $35 \%$ choose the empty message. This is a significant difference in communication behavior on the $p<0.05$ level, as can be seen from the logistic regressions in Table 7, which take the weakly balanced network as the baseline category. ${ }^{40}$ Moreover, experts send a truthful message more frequently in the strongly balanced network, where sincere behavior is an efficient equilibrium, than in the weakly balanced network $(p<0.01)$, where it is an inefficient equilibrium.

Result 2. Most experts play sincere. Experts significantly more often play sincere in the strongly balanced than in the weakly balanced and more often in the weakly balanced than in the star network.

Result 2 is consistent with the comparative statics of our equilibrium analysis. ${ }^{41}$

To further analyze how experts condition their communication behavior on the network structure, we inspect heterogeneity among individual participants. We compute for every subject the relative frequency of playing sincere when being a sender (i.e., having a link) in a given treatment and compare treatments by Wilcoxon signed-ranks tests. The tests corroborate Result 2 that senders are more often sincere the more balanced the network is (lower panel of Table C.1.1 in Appendix C.1). In particular, around 32\% of the senders in the star network never choose the sincere strategy profile (second column of Table C.1.1 in Appendix C.1). The fact that in 73\% of these latter instances the sender's signal determines her vote and the empty message is chosen is an indication that these experts actively target the LTED equilibrium. ${ }^{42}$

Still, most of the time experts play sincere, independent of the communication structure. This does not necessarily imply that those experts never target the LTED equilibrium; it might also mean that they intentionally delegate equilibrium selection to the non-experts. ${ }^{43}$

Non-experts. Non-experts in our experiments frequently receive vote recommendations. Apart from the empty network, every non-expert is linked to an expert sender, who in $81 \%$ of the instances sends a non-empty message. The equilibrium analysis of our model showed that the vote recommendation of an expert should only be followed if this expert is not "too powerful" (in terms of audience size). More precisely, the sincere strategy profile $\hat{\sigma}$ in which all non-experts follow their messages is an equilibrium in the strongly and weakly balanced networks of our experiment, but not in the star network. As displayed in Fig. 6 (and in Table 8 in column 'vote message'), in around 70\% to 80\% of the instances non-experts vote

\footnotetext{
39 There are also some deviations in the voting behavior as can be seen from Table 6. For instance, 5.9\% of experts vote against their signal in the empty network, which is a relatively high error rate when compared to the study of Morton and Tyran (2011). However, we note important differences between these two studies including that the experts in our experiment have a lower signal quality than the non-experts (!) in their treatments and that the groups in our study are three times larger.

40 We use robust standard errors clustered by sessions in every regression. All results would also hold when clustering standard errors by subject (as reported in an earlier working paper version).

41 When investigating learning effects by restricting attention to the second half of rounds, it turns out that Result 2 is robust (significant albeit on a weaker significance level) and that experts send their signal less often in the weakly balanced and the star network than in the first half of rounds (going down to around $50 \%$ in the star network). This can be interpreted as them learning to target the efficient equilibrium LTED $\sigma^{*}$.

42 This interpretation is further corroborated by an ex-post questionnaire on the subjects' motivations.

43 There are other, behavioral, reasons for why most experts play sincere even if the network is unbalanced. One might be lying aversion which is common in laboratory experiments. Not sending a message or sending a message that contradicts the own signal might "feel like" lying. Another might be status-seeking behavior by sending messages in order to be influential.
} 


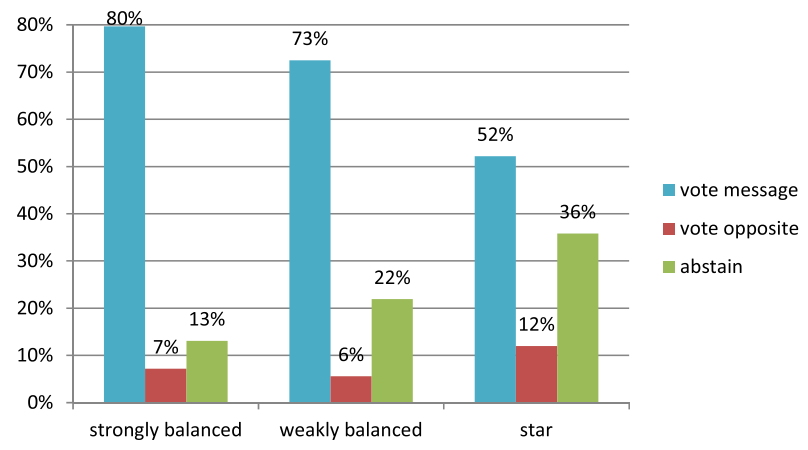

Fig. 6. Frequency of non-experts' following behavior by treatment. 'Vote message' means to vote $A(B)$ when the message received is $A(B)$. 'Vote opposite' means to vote $A(B)$ when the message received is $B(A)$. Only responses to non-empty messages are displayed. Differences between the (strongly and weakly) balanced networks and the star network are significant on the $p<0.01$ level (cf. Table 9).

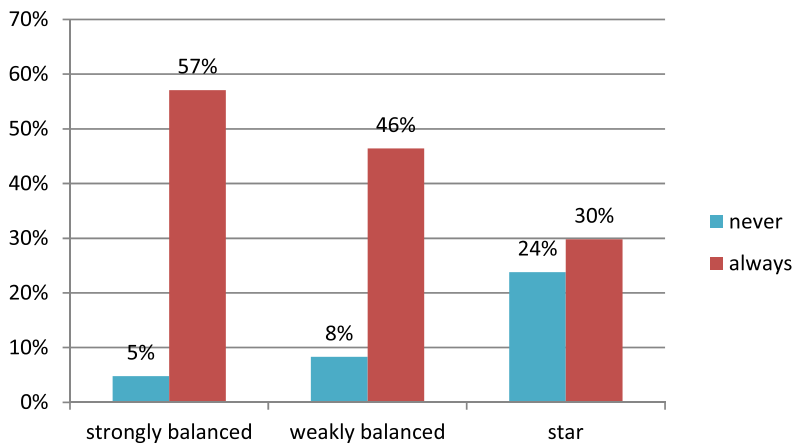

Fig. 7. Frequency of individual following behavior by treatment. The variable 'never', respectively 'always,' reports the fraction of individual participants who never respectively always followed the non-empty vote recommendation they received for each network position. Differences between the (strongly and weakly) balanced networks and the star network are significant on the $p<0.01$ level (cf. Table 10).

according to their received message in the balanced networks where the sincere strategy profile is an equilibrium, but they do so only in around $50 \%$ of the instances in the star network. These differences are significant on the $p<0.01$ level as can be seen from the logistic regressions in Table 9.

Result 3. Non-experts follow their vote recommendation significantly more often in the (strongly and weakly) balanced networks than in the star network.

In general, non-experts who do not follow a message mostly abstain, as can be seen in Fig. 6. Thus, the flipside of a significant decrease in followers is a significant increase in abstentions for the unbalanced networks. ${ }^{44}$

To get more detailed evidence on when non-experts follow their vote recommendations, we move on to idiosyncratic heterogeneity between participants. Fig. 7 shows how many of the non-experts never and how many always follow their message in a given position. As many as $57 \%$ of the non-experts always follow their message when they are in the strongly balanced network, respectively the weakly balanced network. For the star network this number reduces to $30 \%$. This observation strongly suggests that a considerable share of non-experts react to the balancedness of the network, as predicted by our theory ${ }^{45}$ Differences in individual behavior of non-experts across networks are tested using Wilcoxon signed-ranks tests, which are reported in Table 10. In the star network the non-experts' following behavior is different from their be-

\footnotetext{
44 When exploring learning effects by focusing on the second half of rounds, we first of all find that Result 3 is robust. Moreover, we find that following message $A$ or $B$ is less frequent in the second half of rounds than in the first half. In the star network, abstention of non-experts increases (from $37 \%$ to $56 \%$ ), while uninformed voting decreases (from $49 \%$ to $26 \%$ ). This change of behavior is consistent with individual improvements in taking the swing voter's curse into account in the second half.

45 In fact, the ex-post questionnaire with which we asked the subjects about their motives for their decisions corroborates the view that many subjects understood the swing voter's curse in some form. For instance, there was substantial agreement (80\% agree, $7 \%$ agree partially, $13 \%$ disagree) with the following (sound) statement about pivotality reasoning: "With my vote, I have tried to make a good choice for the case that my vote is decisive." There was substantial disagreement ( $26 \%$ agree, $14 \%$ agree partially, $59 \%$ disagree) with the following unsound statement: "The more receivers a certain sender has, the more her message should be followed." Moreover, agreement to the statements is associated with corresponding actual behavior, e.g. agreement to
} 
havior in all other networks ( $p<0.01$, upper block of Table 10). Hence, the network structure has a strong influence on following: a substantial fraction of individuals only follows the vote recommendation of senders that are not too influential. ${ }^{46}$ Besides, there is also a minority of subjects who follow any sender. ${ }^{47}$ In sum, non-experts' behavior is largely in line with the predictions of our model.

\subsection{Efficiency}

Since uninformed voting is highly detrimental to efficiency in common-interest voting (see, e.g., Elbittar et al., 2016), a few remarks on uninformed voting are in order before we proceed to our efficiency results.

Uninformed voting. Non-experts who receive no message, either because they are in the empty network or because their sender chose the empty message, are uninformed. In most of these instances the uninformed non-experts abstain, but in a substantial fraction of around $30 \%$ of instances there is a vote by the uninformed non-expert, as can be seen from Table $8 .{ }^{48}$ This observation is in line with the literature, since positive rates of uninformed voting are found in all experiments on common-interest voting. ${ }^{49}$ Since uninformed votes are no better than coin flips, they have detrimental effects on informational efficiency. However, if we compute the hypothetical group decisions without uninformed voting (as if all uninformed voters abstained), then only 14 out of 840 decisions, i.e., less than $2 \%$, change: In 8 instances a majority for $A$ or $B$ changes into a tie; and in 6 instances a tie changes into a majority for $A$ or $B$. Hence, the impact of uninformed voting on efficiency in our study is limited.

Informational efficiency. Per definitionem, informational efficiency is the higher the more often the signal received by the majority of experts determines the voting outcome. Given the results on individual behavior and equilibrium selection above, we have two opposite forces. Some subjects play the sincere strategy profile $\hat{\sigma}$ independent of the network structure, which is the more inefficient, the less balanced the network. Others mitigate the inefficiency of the unbalanced networks, but not necessarily of the weakly balanced network, by playing a strategy that targets the LTED equilibrium $\sigma^{*}$. The net effect of the two forces is a priori unclear.

Fig. 8 displays the degree of informational efficiency of voting outcomes across networks. To test whether differences in informational efficiency across networks are significant, we create the variable efficiency that takes the value -1 if the voting outcome matches the minority signal, the value 0 if a tie occurs, and the value 1 if the voting outcome matches the majority signal. When Fisher exact tests directly compare efficiency of the different treatments, the null hypothesis cannot be rejected (Table 11). This indicates that there are no strong differences in efficiency between the treatments.

Note, however, that efficiency is also heavily affected by signal distributions. ${ }^{50}$ By controlling for them, we can reduce the noise in the analysis of efficiency. Using ordered logit models, we regress efficiency on the network type, controlling for the signal distribution. Results are displayed in Table 12. We now find weak evidence that informational efficiency is lower in the star network than in the empty network $(p<0.1)$.

Result 4. Informational efficiency is lower in the star network, than in the empty network (weak evidence).

\footnotetext{
the latter statement is highly correlated with following vote recommendations in the star network; agreement to the former statement is correlated with higher expected payoffs.

46 As an anonymous referee has pointed out, our finding that non-experts target the sincere equilibrium more often than the LTED equilibrium in the weakly and strongly balanced networks, compared to the star, might also be due to homogeneous signal qualities among experts. If experts were of different quality as in our general model, voters might refrain from following the recommendation of low-quality experts to delegate the decision to the high-quality experts.

47 There are at least three possible explanations of their behavior. One is extreme correlation neglect (see, e.g., Enke and Zimmermann, 2017, and Levy and Razin, 2015): These non-experts might ignore the perfect correlation of a message received by several members of an expert's audience, instead treating it as an independent signal. The second possible explanation is overconfidence in the truth of the signal of one's own sender (see, e.g., Ortoleva and Snowberg, 2015). The third is what one might call "pivotality neglect", i.e., the inability to condition on hypothetical events such as pivotality (see Esponda and Vespa, 2014). Obviously, also a combination of biases might explain the amount of naïve following. For instance, if a non-expert linked to the center of the star in Study I believes that all other voters randomize and that he himself is likely to be pivotal, his optimal response is indeed to vote along with the message he received (see Dittmann et al., 2014, for a combination of illusion of control, overconfidence, and overestimation of others' errors explaining excess turnout). Since our focus in this paper is not on disentangling biases that lead to irrational voting, we leave a more precise explanation of this minority behavior to further research.

48 Exploring individual heterogeneity in uninformed voting reveals that around $50 \%$ of the participants never vote when uninformed, while there are almost $20 \%$ of the participants who always vote when uninformed.

49 Grosser and Seebauer (2016) find a 30\% rate of uninformed voting. Elbittar et al. (2016) even find that 60\% of the uninformed vote.

50 To see this, let the five experts happen to receive the same signal, say $A^{*}$. They will find it is easier to implement the majority signal $A^{*}$ than if there were signals for both $A$ and $B$, where voting errors were more likely to impair informational efficiency. We call a signal distribution of the form " $5: 0$ " uniform, a signal distribution of the form " $4: 1$ " almost uniform, and a signal distribution of the form "3:2" non-uniform.
} 


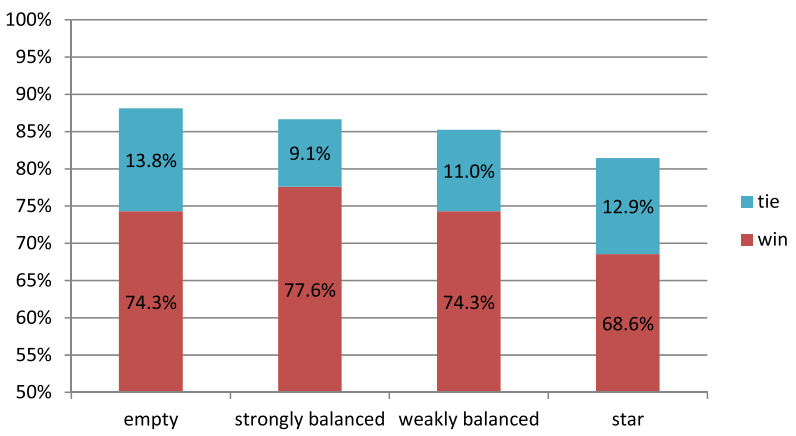

Fig. 8. Frequency of informationally efficient group decisions by treatment. 'Win' means that the outcome of voting is the majority signal. 'Tie' means that there were as many votes for $A$ as for $B$ such that the outcome is correct with probability one half. Differences between the empty network and the star network are significant on the $p<0.1$ level when controlling for the distribution of signals (Table 12).

The superiority of the empty network compared to the star network is so notable because any strategy profile that can be played in the empty network is also feasible in this unbalanced network. Providing participants with the possibility to communicate can hence have a detrimental effect on their voting outcome. ${ }^{51}$

Economic efficiency. To test whether the low informational efficiency in the star network, and probably also in the unbalanced network, affects subjects in an economically meaningful way, we compute the expected payoff $E P$ for each group in each round. If the group decision matches the true state, each member of the group earns 100 points. Hence, the variable $E P$ coincides with the likelihood (in percentage points) of a correct collective decision, given all signals in the group. For instance, if four experts have received signal $A^{*}$ and one expert $B^{*}$ and the outcome of the majority vote is $A$, then $E P=\frac{p^{4}(1-p)}{p^{4}(1-p)+(1-p)^{4} p} * 100$ which is approximately 77.14 for $p=0.6 .{ }^{52}$ Computing $E P$ by network type yields on average 61 points in the star network and on average 64 points in the other networks (as displayed in Table C.1.2 in Appendix C.1). ${ }^{53}$

Recall that when not controlling for the distribution of signals, there is additional noise because some treatments might happen to exhibit uniform signals and hence higher expected payoffs more often than others. We test for significant differences using OLS regressions and control for uniformity of signals (Table C.1.3). The findings are similar to those of Result 4: The inefficiency of the star network compared to the empty network is confirmed $(p<0.1)$. In addition, there is now weak evidence that the star network exhibits lower efficiency than the weakly balanced network $(p<0.1)$.

Result 5. Expected payoffs are lower in the star network than in the empty network and the weakly balanced network (weak evidence).

Result 5 consists of two separate findings. The comparison among the networks in which communication is possible shows that an unbalanced communication structure can be detrimental to efficiency. The comparison of the star network with the empty network, where communication is precluded, shows that communication itself can be detrimental to efficiency, confirming Result 4 above. ${ }^{54,55}$

To summarize, although we find evidence in favor of the comparative statics of our theory and a substantial share of our subjects does switch from sincere voting to the LTED equilibrium if network balancedness decreases, this switching behavior is not pronounced enough to fully prevent detrimental effects of unbalanced communication on informational efficiency. If instead, preplay communication is prohibited altogether, voters can indeed be better off. Despite these deviations, our experimental results confirm that our simple model already captures an important aspect of pre-vote communication in social networks: Balancedness of the network structure affects both the choice between sincere behavior and LTED, and efficiency.

\footnotetext{
51 When exploring learning effects by focusing on the second half of rounds, we first of all find that Result 4 is robust. Moreover, it yields in addition weak evidence $(p<0.1)$ that the star is less efficient than the weakly balanced network. Hence, informational inefficiency does not vanish when agents have time to learn.

52 If we consider reasonable values of $E P$ to lie between the $E P$ of a dictator who is randomly chosen from the experts and the $E P$ of an efficient strategy profile, then the range is $[60,68.3]$.

53 Table C.1.2 additionally displays the actual number of correct group decisions ('success'), which is a less reliable measure of economic efficiency than $E P$ due to the noise induced by imperfect signals. As confirmed by $t$-tests (not in the appendix) the empirical values of $E P$ are significantly below the $E P$ of an efficient strategy profile, except for the case of a uniform signal, i.e., a signal distribution of the form " $5: 0$," which virtually always leads to the efficient majority decision.

54 When exploring learning effects by focusing on the second half of rounds, we cannot reject the null hypothesis that expected payoffs in the different treatments are the same. However, for the empty network, there is weak evidence that in the second half of rounds expected payoffs are larger than in the first half $(p<0.1)$. There are no similar learning effects in the other treatments.

55 Further empirical results show that most inefficient outcomes could have been avoided by some non-expert voters abstaining (see Table C.1.4 in SOM C.1).
} 


\section{Conclusion and discussion}

Basic model. We have analyzed communication in the form of vote recommendations in social networks and have shown that it can lower efficiency of the voting outcome even in a common-interest setting. In the basic model communication is fully private: The network is bipartite and the non-experts have at most degree one. After communication, each voter holds no more than one piece of information. Hence, any information aggregation can only occur in the voting stage, not through communication.

If such a social network is not strongly balanced, i.e., if it gives too much weight to voters with too little expertise, and if, at the same time, the network is not unbalanced either, then it can be an equilibrium to give and follow vote recommendations. However, the outcome of the resulting election is less likely to be optimal than under no communication. We have compared two focal strategy profiles - one without and one with private vote recommendations. In the first, efficient strategy profile, the informed vote in line with their own information and the others abstain ("let the experts decide"). In the second strategy profile, voters report their information to their neighbors in the network and everyone votes according to his or her updated belief (sincere behavior). Theoretically, we have found that sincere behavior, i.e., giving and following vote recommendations, constitutes an equilibrium in many weakly balanced networks but is inefficient in all that are not strongly balanced. We have shown empirically that the two focal strategy profiles are indeed the only relevant ones, that the inefficient one is frequently chosen over the efficient one, and that communication indeed has some detrimental effects on efficiency if the network is unbalanced.

General model. In Appendix A.2, we generalize our model to include vote recommendations between arbitrary voters with varying levels of expertise and in arbitrary network structures, but still in a common-interest setting. The general model includes both extremes of the private-public communication spectrum as special cases. Fully public communication occurs if the network is complete, i.e., every voter is linked to every other voter. In this case, passing on one's signal and voting according to one's posterior belief (sincere behavior) is efficient and an equilibrium that captures ideal deliberation: The optimal alternative can be deduced by every voter; and other voting rules than the majority rule would also admit an equally efficient outcome (cf. Coughlan, 2000, Austen-Smith and Feddersen, 2006, and Gerardi and Yariv, 2007).

Arguably, in large elections communication is neither fully public nor fully private. Receiving multiple messages and an own signal leads to some information aggregation already in the communication stage. Participation in a majority election further aggregates information in the voting stage. Our general model accounts for this reality with respect to vote recommendations. Balancedness is crucial here, too: With the exception of efficiency of LTED in finite networks, the findings from our basic model remain robust. Moreover, all findings from the basic model also hold in the limit when the population size converges to infinity.

To be clear, also the general version of our model addresses only one form of pre-vote communication: vote recommendations. This is captured by our set-up and the particular communication protocol, in which there is one round of sending messages to all network neighbors.

Misinformation. Our setting lends itself to be extended to study the effects of misinformation on voting behavior and informational efficiency. We did one step into this direction which is reported in SOM C.3 and in an earlier working paper version that is permanently available online (Buechel and Mechtenberg, 2017). In Buechel and Mechtenberg (2017), we extend the simple model from section 2 of this paper to include "partisans" (Feddersen and Pesendorfer, 1996), i.e., troll senders that either always send the message $A$ or always send the message $B$. We assume an equal number of troll senders for both messages. Our equilibrium analysis reveals that the presence of these troll senders does not change our theoretical results: Troll senders reduce the expected quality (i.e., truth probability) of a message, but since our results hold for any expected message quality above 0.5 , this does not add any new insight. Empirically, however, the presence of troll senders creates a new behavioral effect: When we test the extended simple model in the laboratory (Study II, summarized in SOM C.3), we find suggestive evidence that (1) the presence of troll senders makes human senders prone to pass on their signal to their audience regardless of the network structure, and that (2) the star network becomes even less efficient, compared to balanced networks, mostly due to followers of a troll sender in the center of the star. While this is obviously not a comprehensive analysis of misinformation, it still provides an important insight: Misinformation might enlarge an already existing problem of vote recommendations in social networks, namely the too-high weight that voters with large audiences get in the course of communication via social media. ${ }^{56}$

We conclude that the structure of social networks and the balance - or the lack thereof - between voters' expertise and the size of their audience in the communication network are important for efficient majority votes.

\section{Declaration of competing interest}

None.

\footnotetext{
${ }^{56}$ For a recent experimental study on misinformation, see Pogorelskiy and Shum (2019).
} 


\section{Appendix A. Tables and proofs}

\section{A.1. Tables 2-12}

Table 2

Observations.

\begin{tabular}{lllll}
\hline Treatment & Groups & Experts & Non-experts & Receivers \\
\hline empty & 210 & 1,050 & 840 & 0 \\
strongly balanced & 210 & 1,050 & 840 & 840 \\
weakly balanced & 210 & 1,050 & 840 & 420 \\
star & 210 & 1,050 & 840 & 210 \\
Total & 840 & 4,200 & 3,360 & 840 \\
\hline
\end{tabular}

Notes: Number of observations. Senders are experts who are in a network position with an audience. Non-experts are receivers if they are linked to a sender.

Table 3

Strategy profiles.

\begin{tabular}{|c|c|c|c|c|c|}
\hline & LTED $\sigma^{*}$ & Almost LTED & Sincere $\hat{\sigma}$ & Almost sincere & Explained \\
\hline empty $(N=210)$ & $11.0 \%$ & $41.4 \%$ & & & $81.9 \%$ \\
\hline strongly balanced $(N=210)$ & $0.0 \%$ & $2.4 \%$ & $10.0 \%$ & $35.2 \%$ & $81.0 \%$ \\
\hline weakly balanced $(N=210)$ & $1.0 \%$ & $5.7 \%$ & $7.6 \%$ & $27.6 \%$ & $82.9 \%$ \\
\hline $\operatorname{star}(N=210)$ & $2.4 \%$ & $12.4 \%$ & $2.4 \%$ & $13.8 \%$ & $90.5 \%$ \\
\hline Total $(N=840)$ & $3.6 \%$ & $15.5 \%$ & $6.7 \%$ & $25.6 \%$ & $84.0 \%$ \\
\hline
\end{tabular}

Notes: Frequency of strategy profiles. A group plays "almost" a strategy profile if there is at most one player whose strategy differs from the profile. An outcome is explained if the actual outcome in a group (i.e., majority decision $A$ or $B$ ) is predicted by at least one of the two strategy profiles, given the distribution of signals.

\section{Table 4}

Fisher exact tests on (almost) $\sigma^{*}$.

\begin{tabular}{llll}
\hline & Strongly balanced & Weakly balanced & Star \\
\hline empty & $0.000(0.000)$ & $0.000(0.000)$ & $0.001(0.000)$ \\
strongly balanced & & $0.499(0.135)$ & $0.061(0.000)$ \\
weakly balanced & & & $0.449(0.026)$ \\
\hline
\end{tabular}

Notes: $p$-values of Fisher exact tests comparing the frequency of the "LTED" strategy profile $\sigma^{*}$ between two treatments. In brackets are the corresponding $p$-values for almost LTED $\sigma^{*}$. A group plays "almost" $\sigma^{*}$ if there is at most one player whose strategy differs from the profile.

Table 5

Fisher exact tests on (almost) $\hat{\sigma}$.

\begin{tabular}{|c|c|c|}
\hline & Weakly balanced & Star \\
\hline strongly balanced & $0.492(0.115)$ & $0.002(0.000)$ \\
\hline weakly balanced & & $0.023(0.001)$ \\
\hline
\end{tabular}

Table 6

Behavior of experts.

\begin{tabular}{|c|c|c|c|c|c|}
\hline & Vote signal & Vote opposite & Send signal & Send opposite & Sincere \\
\hline empty & 919 & 62 & - & - & 919 \\
\hline$(N=1,050)$ & $87.5 \%$ & $5.9 \%$ & - & - & $87.5 \%$ \\
\hline strongly balanced & 884 & 75 & 671 & 53 & 798 \\
\hline$(N=1,050)$ & $84.2 \%$ & $7.1 \%$ & $79.9 \%$ & $6.3 \%$ & $76.0 \%$ \\
\hline weakly balanced & 878 & 74 & 301 & 30 & 803 \\
\hline$(N=1,050)$ & $83.6 \%$ & $7.1 \%$ & $71.7 \%$ & $7.1 \%$ & $76.5 \%$ \\
\hline star & 854 & 84 & 128 & 9 & 794 \\
\hline$(N=1,050)$ & $81.3 \%$ & $8.0 \%$ & $61.0 \%$ & $4.3 \%$ & $75.6 \%$ \\
\hline Total & 3,535 & 295 & 1,100 & 92 & 3,314 \\
\hline$(N=4,200)$ & $84.2 \%$ & $7.0 \%$ & $74.8 \%$ & $6.3 \%$ & $78.9 \%$ \\
\hline
\end{tabular}

Notes: Behavior of experts by treatment. The action 'vote (send) opposite' means vote (send message) $A$ when signal is $B^{*}$ and vice versa. In addition to the displayed categories 'vote signal' and 'vote opposite' experts could abstain. In addition to the displayed categories 'send signal' and 'send opposite' experts could send an empty message. Experts without an audience are sincere if they vote their signal. Experts with an audience are sincere if they vote their signal and also send it. 
Table 7

Sincere senders.

\begin{tabular}{|c|c|c|c|c|}
\hline \multirow[t]{2}{*}{ Variable } & \multicolumn{2}{|c|}{ Logit 1: Send Signal } & \multicolumn{2}{|c|}{ Logit 2: Sincere } \\
\hline & Coefficient & (Std. Err.) & Coefficient & (Std. Err.) \\
\hline strongly balanced & $0.451^{* * *}$ & $(0.175)$ & $0.505^{* * *}$ & $(0.115)$ \\
\hline star & $-0.483^{* *}$ & $(0.191)$ & $-0.486^{* * *}$ & $(0.159)$ \\
\hline Intercept & $0.928^{* * *}$ & $(0.189)$ & $0.619^{* * *}$ & $(0.122)$ \\
\hline$N$ & \multicolumn{2}{|c|}{1,470} & \multicolumn{2}{|c|}{1,470} \\
\hline Log-likelihood & \multicolumn{2}{|c|}{-812.55} & \multicolumn{2}{|c|}{-884.94} \\
\hline Wald $\chi_{(2)}^{2}$ & \multicolumn{2}{|c|}{9.33} & \multicolumn{2}{|c|}{19.24} \\
\hline$p$-value Wald test & \multicolumn{2}{|c|}{0.009} & \multicolumn{2}{|c|}{0.000} \\
\hline
\end{tabular}

Notes: Estimation results: Logistic regression sincere senders by treatment. Senders are experts with at least one link Dependent variable in Model 1 is 'send signal,' which is 1 if the expert's message equals her signal (and zero otherwise). Dependent variable in Model 2 is sincere behavior, which equals 1 if sender both sends and votes her signal. Robust standard errors in parentheses adjusted for sessions. Baseline category is the weakly balanced network.

${ }^{*} p<0.10,{ }^{* *} p<0.05,{ }^{* * *} p<0.01$.

Table 8

Behavior of non-experts.

\begin{tabular}{lllll}
\hline & Vote message & Vote opposite & Vote uninformed & Sincere \\
\hline empty & - & - & 265 & 575 \\
$(N=840)$ & - & - & $31.6 \%$ & $68.5 \%$ \\
strongly balanced & 577 & 52 & 29 & 664 \\
$(N=840)$ & $79.7 \%$ & $7.2 \%$ & $25.0 \%$ & $79.1 \%$ \\
weakly balanced & 480 & 37 & 49 & 709 \\
$(N=840)$ & $72.5 \%$ & $5.6 \%$ & $27.5 \%$ & 470 \\
star & 286 & 66 & 108 & $56.0 \%$ \\
$(N=840)$ & $52.2 \%$ & $12.0 \%$ & $37.0 \%$ & 2,318 \\
Total & 1,343 & 155 & 451 & $69.0 \%$ \\
$(N=3,360)$ & $69.4 \%$ & $8.0 \%$ & $31.6 \%$ & \\
\hline
\end{tabular}

Notes: Behavior of non-experts by treatment. In the empty network all non-experts are uninformed. In the other networks this happens only if an expert sender chose the empty message. The action 'vote message' means that $A(B)$ is voted after message $A(B)$ has been received. In addition to the displayed categories 'vote message' and 'vote opposite' non-experts who received message $A$ or $B$ could abstain. In addition to the displayed category 'vote uninformed' non-experts who received an empty message could abstain. Non-experts with no message or an empty message are sincere if they abstain. Non-experts with message $A(B)$ are sincere if they vote $A(B)$.

\section{Table 9}

Dependent variable: Following of non-experts.

\begin{tabular}{|c|c|c|c|c|}
\hline \multirow[t]{2}{*}{ Variable } & \multicolumn{2}{|l|}{ Logit 1} & \multicolumn{2}{|l|}{ Logit 2} \\
\hline & Coefficient & (Std. Err.) & Coefficient & (Std. Err.) \\
\hline strongly balanced & 0.398 & $(0.266)$ & & \\
\hline weakly balanced & & & -0.398 & $(0.266)$ \\
\hline star & $-0.882^{* * *}$ & $(0.160)$ & $-1.280^{* * *}$ & $(0.195)$ \\
\hline Intercept & $0.970^{* * *}$ & $(0.249)$ & $1.367^{* * *}$ & $(0.272)$ \\
\hline$N$ & \multicolumn{2}{|c|}{1,934} & \multicolumn{2}{|c|}{1,934} \\
\hline Log-likelihood & \multicolumn{2}{|c|}{-1133.96} & \multicolumn{2}{|c|}{-1133.96} \\
\hline Wald $\chi_{(2)}^{2}$ & \multicolumn{2}{|c|}{83.96} & \multicolumn{2}{|c|}{83.96} \\
\hline$p$-value Wald test & \multicolumn{2}{|c|}{0.000} & \multicolumn{2}{|c|}{0.000} \\
\hline
\end{tabular}

Notes: Estimation results: Logistic regression with decision to follow a (non-empty) message as dependent variable. Robust standard errors in parentheses adjusted for sessions. Baseline category is the weakly balanced network in Model 1 and the strongly balanced network in Model 2 . Following coincides with sincere behavior of non-experts who have received message $A$ or $B$.

${ }^{*} p<0.10,{ }^{* *} p<0.05$, *** $p<0.01$.

Table 10

Individual sincere behavior of non-experts.

\begin{tabular}{lllll}
\hline & Never & Always & Strongly balanced & Weakly balanced \\
\hline strongly balanced & $4.8 \%$ & $57.1 \%$ & & 0.127 \\
weakly balanced & $8.33 \%$ & $46.43 \%$ & & 0.000 \\
star & $23.8 \%$ & $29.8 \%$ & 0.000 \\
empty & $19.1 \%$ & $54.8 \%$ & 0.033 & 0.545 \\
strongly balanced & $1.2 \%$ & $51.2 \%$ & & 0.008
\end{tabular}


Table 10 (continued)

\begin{tabular}{llll}
\hline & Never & Always & Strongly balanced \\
\hline weakly balanced & $0.0 \%$ & $31.0 \%$ & Weakly balanced \\
star & $1.2 \%$ & $16.7 \%$ & 0.000 \\
\hline
\end{tabular}

Notes: Individual behavior of non-experts: for each individual there is a variable capturing the frequency of sincere actions. The first block restricts attention to instances in which a non-empty message is received and thus reports on following of non-empty vote recommendations. The second block considers all ten decisions of each individual in each network. In the empty network a non-expert never receives a message. Column 2 and 3 report the fraction of participants who never respectively always chose the sincere strategy in the given network. Columns 4-6 of the table show the $p$-values of Wilcoxon matched-pairs signed-ranks test.

Table 11

Fisher exact tests on efficiency.

\begin{tabular}{llll}
\hline & Strongly balanced & Weakly balanced & star \\
\hline empty & 0.299 & 0.543 & 0.170 \\
strongly balanced & & 0.705 & 0.117 \\
weakly balanced & & 0.429 \\
\hline
\end{tabular}

Notes: $p$-values of Fisher exact tests comparing efficiency between two treatments. Efficiency is 1 if majority signal wins, 0 in case of a tie, and -1 if majority signal loses.

Table 12

Dependent variable: Efficiency.

\begin{tabular}{|c|c|c|c|c|}
\hline \multirow[t]{2}{*}{ Variable } & \multicolumn{2}{|l|}{ Ologit 1} & \multicolumn{2}{|l|}{ Ologit 2} \\
\hline & Coeff. & (Std. Err.) & Coeff. & (Std. Err.) \\
\hline empty & & & -0.016 & $(0.185)$ \\
\hline strongly balanced & 0.110 & $(0.265)$ & 0.095 & $(0.238)$ \\
\hline weakly balanced & 0.016 & $(0.185)$ & & \\
\hline star & $-0.236^{*}$ & $(0.141)$ & -0.252 & $(0.174)$ \\
\hline uniform signal & $3.173^{* * *}$ & $(0.593)$ & $3.173^{* * *}$ & $(0.593)$ \\
\hline almost uniform signal & $1.579^{* * *}$ & $(0.367)$ & $1.579^{* * *}$ & $(0.367)$ \\
\hline Intercept cut 1 & -1.296 & $(0.110)$ & -1.311 & $(0.152)$ \\
\hline Intercept cut 2 & -0.492 & $(0.121)$ & -0.508 & $(0.126)$ \\
\hline$N$ & \multirow{2}{*}{\multicolumn{2}{|c|}{$\begin{array}{c}840 \\
580.612\end{array}$}} & \multicolumn{2}{|c|}{840} \\
\hline Log-likelihood & & & \multicolumn{2}{|c|}{-580.612} \\
\hline
\end{tabular}

Notes: Estimation results: Ordered logit. Efficiency is 1 if majority signal wins, 0 in case of a tie, and -1 if majority signal loses. Robust standard errors in parentheses adjusted for sessions. Less clusters than parameters simply mean that joint significance (Wald test) cannot be tested. The first model uses the empty network as baseline category. The second model uses the weakly balanced network as baseline category.

${ }^{*} p<0.10,{ }^{* *} p<0.05,{ }^{* * *} p<0.01$.

\section{A.2. General model and proofs}

We now take the model as it is defined in section 2 and integrate the following two extensions. First, we relax the assumptions that only experts receive signals and that all experts' signals are of equal quality $p$. Instead we assume that every agent $i$ receives a signal with idiosyncratic signal quality $p_{i} \in\left[\frac{1}{2}, 1\right)$. Second, we relax the assumptions on the network structure. We now admit arbitrary network structures in which agents can receive multiple messages and be informed by nature in addition.

\section{A.2.1. Set-up}

As before, nature draws one state of the world $\omega \in\{A, B\}$ with uniform probability. There is a finite set of voters $V$. All agents $i \in V$ receive a private independent signal $s_{i} \in\left\{A^{*}, B^{*}\right\}$ about the true state of the world with quality $p_{i}=$ $\operatorname{Pr}\left\{s_{i}=A^{*} \mid \omega=A\right\}=\operatorname{Pr}\left\{s_{i}=B^{*} \mid \omega=B\right\} \in\left[\frac{1}{2}, 1\right)$. Let $g^{V}$ be the set of all subsets of $V$ of size two. A network $g \subseteq g^{V}$ represents the communication structure between the agents. A voter of degree $d_{i} \geq 1$ is called sender and all voters linked to her are called her neighbors, who are denoted by $V_{i}:=\{j \in V \mid i j \in g\}$.

The voting stage and the preferences are as defined in section 2. In particular, after receiving the signal, each agent may send message "A" or message "B" or an empty message $\emptyset$ to her neighbors. Then, all agents simultaneously participate in the majority vote. Note that senders may be neighbors of other senders now. Strategies can now be defined as follows: A communication strategy $m_{i}$ of a voter $i \in V$ with $d_{i} \geq 1$ defines which message to send for each signal received, i.e. $m_{i}:\left\{A^{*}, B^{*}\right\} \rightarrow\{A, B, \varnothing\}$. A voting strategy $v_{i}$ of a voter $i \in V$ defines whether and how to vote for each signal received and for each profile of messages received, i.e. $v_{i}:\left\{A^{*}, B^{*}\right\} \times\{A, B, \varnothing\}^{d_{i}} \rightarrow\{A, B, \emptyset\}$ if $d_{i} \geq 1$ and $\sigma_{j}:\left\{A^{*}, B^{*}\right\} \rightarrow\{A, B, \emptyset\}$ if $d_{j}=0$. Again, we denote by $\sigma_{i}=\left(m_{i}, v_{i}\right)$ a communication and voting strategy of a voter and by $\sigma=\left(\sigma_{i}\right)_{i \in V}$ a strategy profile. $^{57}$

57 This model nests our model of section 2 as follows. Let experts $M \subseteq V$ be a subset of voters who receive an informative signal of quality $p_{j}=p>\frac{1}{2}$ and let $N=V \backslash M$ be the non-experts, whose signal is uninformative, i.e. $p_{i}=\frac{1}{2}$. Moreover, we assumed that $g$ is bipartite such that all links involve exactly one expert and one non-expert and that non-experts have at most one link. 
In the general model the definition of experts and non-experts has to be reconsidered. Order the voters by their signal quality in decreasing order such that $p_{1} \geq p_{2} \geq \ldots \geq p_{|V|}$ (in case of equalities fix any such order) and consider the $m$ best informed voters as the experts, i.e., set $M:=\left\{j_{1}, j_{2}, \ldots, j_{m}\right\} \subseteq V$ with $p_{m}>\frac{1}{2}$. Consistently, the LTED strategy profile $\sigma^{*, m}$ will be parametrized by the number of experts $m$, which we require to be odd. ${ }^{58}$

In the simple model with homogeneous signal precision, the "expertise" of a set of voters could be assessed simply by the number of signals they have received, which is the number of experts in the set. For idiosyncratic signal precision, each signal must be considered with its quality $p_{i}$. The optimal aggregation rule weights each signal with its "log-odds" weight $\log \left(\frac{p_{i}}{1-p_{i}}\right)$ (Nitzan and Paroush, 1982; Shapley and Grofman, 1984). Hence, a set of voters $S$ is better informed than another set $S^{\prime}$ if and only if $\sum_{i \in S} \log \left(\frac{p_{i}}{1-p_{i}}\right)>\sum_{j \in S^{\prime}} \log \left(\frac{p_{j}}{1-p_{j}}\right)$, which simplifies to $\prod_{i \in S} \frac{p_{i}}{1-p_{i}}>\prod_{j \in S^{\prime}} \frac{p_{j}}{1-p_{j}}$.

In this more general set-up the definition of the sincere strategy profile $\hat{\sigma}$ has to be extended since voters may receive multiple messages, while still all voters with an informative signal communicate their signal. Consistent with the tag "sincere" we assume that agents vote for the message that has the higher posterior probability to coincide with the true state, given their private information, i.e., a voter $i$ who has received signal $A^{*}$ from nature and message $A$ by the subset $S$ of her neighbors $V_{i}$ votes for $A$ if and only if $\sum_{j \in S \cup\{i\}} \log \left(\frac{p_{j}}{1-p_{j}}\right)>\sum_{k \in V_{i} \backslash(S \cup\{i\})} \log \left(\frac{p_{k}}{1-p_{k}}\right)$ and abstains if indifferent; and vice versa for a voter who has received signal $B^{*}$. For a voter $j$ who talks to agents with a sufficiently low signal quality (i.e. $\left.\log \left(\frac{p_{j}}{1-p_{j}}\right)>\sum_{k \in V_{i}} \log \left(\frac{p_{k}}{1-p_{k}}\right)\right)$ this simply means to vote in line with her signal. For a voter without an informative signal who listens to some equally well-informed experts, $\hat{\sigma}$ means to vote in line with the message $A$ or $B$ that he has received more often; if both messages have been received equally frequently or if there is no message at all, he abstains. We can now reconsider our three theoretical results, Propositions $2.1-2.3$, in the more general set-up.

\section{A.2.2. "Let the experts decide" revisited}

Propositions 2.1 shows for the particular model that the LTED strategy profile $\sigma^{*}$ is efficient and an equilibrium. In the general model, every odd number $m=1,3,5, \ldots$ that is not larger than the number of informed agents defines a LTED strategy profile $\sigma^{*, m}$, in which all members of $M$ vote their signal and all voters outside of $M$ abstain. We find that each of these strategy profiles is an equilibrium and that efficiency holds at least asymptotically.

Proposition A.1 (LTED, general). There exist equilibria for any network structure. For instance, for any odd number $m$ of experts (i.e., $m$ voters with signal precision strictly above $\frac{1}{2}$ and weakly above all other voters'), the LTED strategy profile $\sigma^{*, m}$ is an equilibrium for any network structure. This strategy profile is efficient if and only if $\prod_{j \in M^{\prime}} \frac{p_{j}}{1-p_{j}} \geq \prod_{k \in V \backslash M^{\prime}} \frac{p_{k}}{1-p_{k}}$, where $M^{\prime}:=\left\{j_{\frac{m+1}{2}}, j_{\frac{m+3}{2}}, \ldots, j_{m}\right\} \subseteq M$ is the set of the $\frac{m+1}{2}$ experts with the lowest signal precision. Moreover, letting the number of experts $m$ grow in this strategy profile $\sigma^{*}, m$, the probability of a correct decision approaches one.

Proof. We address equilibrium, efficiency, and the limit case in turn.

Equilibrium. We first show that $\sigma^{*, m}$ is an equilibrium for any odd $m$. We start with showing that the experts $j \in M$ indeed prefer to vote their signal, then we turn to showing that the non-experts indeed prefer to abstain.

W.l.o.g. consider an expert $j$ who has received signal $A^{*}$. He is pivotal if and only if $A$ wins by one vote ( $m$ is odd). This happens if and only if there is a set of experts $S$ of size $\frac{m-1}{2}$ who have received signal $A^{*}$ as well, while the $\frac{m-1}{2}$ remaining experts $M \backslash(S \cup j)$ have received the signal $B^{*}$. Given that we are in this case ( $j$ has received signal $A^{*}$, all agents in $S$ have received signal $A^{*}$, and all agents in $M \backslash(S \cup j)$ have received signal $\left.B^{*}\right), A$ is weakly more likely to be true than $B$ if and only if

$$
p_{j} \cdot \sum_{S \subset M \backslash j:|S|=\frac{m-1}{2}} \prod_{i \in S} p_{i} \cdot \prod_{k \in M \backslash(S \cup j)}\left(1-p_{k}\right) \geq\left(1-p_{j}\right) \cdot \sum_{Q \subset M \backslash j:|Q|=\frac{m-1}{2}} \prod_{i \in Q}\left(1-p_{i}\right) \cdot \prod_{k \in M \backslash(Q \cup j)} p_{k} .
$$

We observe that for every set " $S$ " in the summation on the LHS of (A.1), there is a complementary set "Q" in the summation on the RHS: $Q=(M \backslash j) \backslash S$. Each pair of "S" and complementary "Q" cancel each other out when computing the products $\prod_{i \in S} p_{i} \cdot \prod_{k \in M \backslash(S \cup j)}\left(1-p_{k}\right)=\prod_{k \in M \backslash(Q \cup j)} p_{k} \cdot \prod_{i \in Q}\left(1-p_{i}\right)$. Therefore, we have

$$
\sum_{S \subset M \backslash j:|S|=\frac{m-1}{2}} \prod_{i \in S} p_{i} \cdot \prod_{k \in M \backslash(S \cup j)}\left(1-p_{k}\right)=\sum_{Q \subset M \backslash j:|Q|=\frac{m-1}{2}} \prod_{k \in M \backslash(Q \cup j)} p_{k} \cdot \prod_{i \in Q}\left(1-p_{i}\right),
$$

which yields that (A.1) holds if and only if $p_{j} \geq 1-p_{j}$, which holds by assumption. Thus, $j \in M$ does not deviate from voting the received signal.

Now, we turn to a non-expert $i \in V \backslash M$. Suppose w.l.o.g. that he has received signal $A^{*}$. Then he is pivotal if and only if $B$ wins by one vote under $\sigma^{*, m}$. This happens if and only if there is a set of experts $S \subset M$ of size $\frac{m-1}{2}$ who has received signal $A^{*}$ and the $\frac{m+1}{2}$ remaining experts $M \backslash S$ have received signal $B^{*}$. Given that we are in this case ( $i$ and all experts in

\footnotetext{
58 The profile $\sigma^{*, m}$ could also be called "let some(!) experts decide.".
} 
$S$ have received signal $A^{*}$ and all experts in $M \backslash S$ have received signal $B^{*}$ ), $A$ is weakly less likely to be true than $B$ if and only if

$$
p_{i} \cdot \sum_{S \subset M:|S|=\frac{m-1}{2}} \prod_{j \in S} p_{j} \cdot \prod_{k \in M \backslash S}\left(1-p_{k}\right) \leq\left(1-p_{i}\right) \cdot \sum_{Q \subset M:|Q|=\frac{m-1}{2}} \prod_{j \in Q}\left(1-p_{j}\right) \cdot \prod_{k \in M \backslash Q} p_{k} .
$$

For every summand $S$ on the LHS there is a summand $Q$ on the RHS which almost coincides but differs in two factors, e.g.

$$
\begin{aligned}
& S: p_{1} p_{2} \ldots p_{\frac{m-1}{2}} \cdot\left(1-p_{\frac{m+1}{2}}\right)\left(1-p_{\frac{m+3}{2}}\right) \ldots\left(1-p_{m}\right) \cdot p_{i} \\
& Q:\left(1-p_{m}\right)\left(1-p_{m-1}\right) \ldots\left(1-p_{\frac{m+3}{2}}\right) \cdot p_{\frac{m+1}{2}} p_{\frac{m-1}{2}} \ldots p_{1} \cdot\left(1-p_{i}\right)
\end{aligned}
$$

differ in the factors $p_{i}$ and $\left(1-p_{i}\right)$ and the factors $p_{\frac{m+1}{2}}$ and $\left(1-p_{\frac{m+1}{2}}\right)$. For a pair $S, Q$ that differs in the factor $p_{k}$ and $\left(1-p_{k}\right)$, besides $p_{i}$ and $\left(1-p_{i}\right)$, let $k(S):=k$ and let $\alpha(S)>0$ be the common part of $S$ and $Q$. Then we can reorganize (A.2) by subtracting the right-hand side (RHS) on both sides and expressing the common and different part of each pair as follows:

$$
\sum_{S \subset M:|S|=\frac{m-1}{2}} \alpha(S) \cdot\left[\left(1-p_{k(S)}\right) \cdot p_{i}-p_{k(S)} \cdot\left(1-p_{i}\right)\right] \leq 0 .
$$

We observe that $\left(1-p_{k(S)}\right) \cdot p_{i} \leq p_{k(S)} \cdot\left(1-p_{i}\right)$ for $p_{i} \leq p_{k(S)}$, which holds by assumption because signal precision of nonexpert $i$ is by definition smaller than of any expert $k(S)$. Thus, (A.3) holds. Hence, for a non-expert $i$ pivotality implies that the outcome of the vote is more likely to be correct than what he can induce with a deviation.

Efficiency. We now show that $\sigma^{*, m}$ is efficient if and only if the following condition holds: $\left(^{*}\right) \prod_{j \in M^{\prime}} \frac{p_{j}}{1-p_{j}} \geq \prod_{k \in V \backslash M^{\prime}} \frac{p_{k}}{1-p_{k}}$, where $M^{\prime}:=\left\{j_{\frac{m+1}{2}}, j_{\frac{m+3}{2}}, \ldots, j_{m}\right\} \subseteq M$ is the set of the $\frac{m+1}{2}$ experts with the lowest signal precision.

For a given draw of nature denote by $S$ the set of agents who have received signal $A^{*}$. Generally, a strategy profile is efficient if and only if the outcome is $A$ whenever $A$ is more likely to be the true state than $B$, i.e., whenever

$$
\sum_{j \in S} \log \left(\frac{p_{j}}{1-p_{j}}\right)>\sum_{k \in V \backslash S} \log \left(\frac{p_{k}}{1-p_{k}}\right)
$$

and the outcome is $B$ whenever inequality (A.4) is reversed (e.g., Shapley and Grofman, 1984, Theorem II).

Suppose, $\sigma^{*, m}$ is efficient. Consider the draw of nature $S=M^{\prime}$, i.e., in which all members of $M^{\prime}$ have received signal $A^{*}$, while all others have received signal $B^{*}$. Since $m^{\prime}>\frac{m}{2}, A$ wins under $\sigma^{*, m}$. By efficiency it must hold that $\sum_{j \in S} \log \left(\frac{p_{j}}{1-p_{j}}\right) \geq$ $\sum_{k \in V \backslash S} \log \left(\frac{p_{k}}{1-p_{k}}\right)$, which is equivalent to $\prod_{j \in M^{\prime}} \frac{p_{j}}{1-p_{j}} \geq \prod_{k \in V \backslash M^{\prime}} \frac{p_{k}}{1-p_{k}}$, i.e., condition ( $\left.{ }^{*}\right)$ is satisfied.

Now suppose condition $\left({ }^{*}\right)$ is satisfied. Since $M^{\prime}$ is the smallest majority of experts with the least expertise, any set $S \supseteq M^{\prime}$ holds more expertise than $M^{\prime}$ such that condition $\left(^{*}\right)$ is equivalent to $\sum_{j \in S} \log \left(\frac{p_{j}}{1-p_{j}}\right) \geq \sum_{k \in V \backslash S} \log \left(\frac{p_{k}}{1-p_{k}}\right)$ if and only if $|M \cap S|>\frac{m}{2}$. Take any draw of nature and denote by $S$ the set of agents who have received signal $A^{*}$. Suppose first that inequality (A.4) holds. Then by $\left({ }^{*}\right),|M \cap S|>\frac{m}{2}$ holds. Under $\sigma^{*, m}$ the outcome is $A$. Suppose the reverse of inequality (A.4) holds. Then by $\left({ }^{*}\right),|M \cap S|<\frac{m}{2}$ holds and $B$ wins. Hence, $\sigma^{*, m}$ is efficient.

Limit. Finally, we show that letting the number of experts $m$ grow in this strategy profile $\sigma^{*, m}$, the probability of an efficient outcome approaches one.

For every $m$, the probability of an efficient outcome under $\sigma^{*, m}$ is larger than in the hypothetical case that every expert in $M$ has signal precision $p_{m}>0.5$ (which is the lowest among the experts) and also votes her signal. For the hypothetical case, the Condorcet Jury theorem applies, showing that the probability of a correct decision approaches one as $m \rightarrow \infty$. Hence, this is also true for $\sigma^{*, m}$.

This result shows robustness of the LTED equilibrium to the two extensions. First, trivially, more general network structures cannot affect an equilibrium which does not involve information transmission. Second, the LTED equilibrium extends to heterogeneous signal precisions. Efficiency of LTED, however, only holds in finite populations if even the weakest majority of experts $\left(M^{\prime}\right)$ holds more expertise than the strongest minority of experts together with all non-experts. This means that efficiency in finite populations requires that the experts' signal quality is not too heterogeneous and that the non-experts' signal quality is sufficiently low, compared to the experts'.

However, we show in the proof of Proposition A.1 (in Appendix A.2) that the potential inefficiency of LTED vanishes when the absolute number of experts $m$ grows large. In that sense not only existence, but also efficiency of LTED extends to the general model. 


\section{A.2.3. Sincere voting revisited}

We now turn to the extension of Propositions 2.2 and hence to the sincere equilibrium $\hat{\sigma}$. The intuition that the sincere equilibrium $\hat{\sigma}$ requires a network structure that balances a group of experts' "expertise" with their "power" fully carries over. We only have to extend the notion of balancedness, which requires some additional notation. ${ }^{59}$

For a fixed set of agents $S \subseteq V$, partition the voters $V$ into believers $V^{+}(S)$, non-believers $V^{-}(S)$, and neutrals $V^{0}(S)$ as follows: $i$ is called a believer of the set $S$, i.e. $i \in V^{+}(S)$, if, from what $i$ can observe in his neighborhood $\left(V_{i} \cup i\right), S$ is better informed than the complementary set, i.e. $\sum_{j \in\left(V_{i} \cup i\right) \cap S} \log \left(\frac{p_{j}}{1-p_{j}}\right)>\sum_{k \in\left(V_{i} \cup i\right) \backslash S} \log \left(\frac{p_{k}}{1-p_{k}}\right)$. Analogously, $i$ is called a non-believer of the set $S$, i.e. $i \in V^{-}(S)$, if, from what $i$ can observe in his neighborhood $\left(V_{i} \cup i\right)$, $S$ is less well-informed than the complementary set, i.e. $\sum_{j \in\left(V_{i} \cup i\right) \cap S} \log \left(\frac{p_{j}}{1-p_{j}}\right)<\sum_{k \in\left(V_{i} \cup i\right) \backslash S} \log \left(\frac{p_{k}}{1-p_{k}}\right)$. Finally, $i$ is called a neutral with respect to the set $S$, i.e. $i \in V^{0}(S)$, if he is neither a believer nor a non-believer, which happens when the above condition holds with equality. In the special case of homogenous signal precision, the number of links into a given set $S$ determines whether an agent is a believer, a non-believer, or a neutral.

Definition A.1 (Balancedness, general).

(a) Given a profile of signal precisions $p_{j}$, a network is called "strongly balanced" if every set of voters which is better informed than the complementary set has more believers than non-believers, i.e. $\forall S \subseteq V, \prod_{j \in S} \frac{p_{j}}{1-p_{j}}>\prod_{k \in V \backslash S} \frac{p_{k}}{1-p_{k}}$ implies $\left|V^{+}(S)\right|>\left|V^{-}(S)\right|$.

(b) For a voter $i \in V$, let $\mathcal{S}_{i}$ collect all sets of voters $S$, of which $i$ is a believer, i.e. $i \in V^{+}(S)$, and which have slightly more believers than non-believers, i.e. $\left|V^{+}(S)\right|-\left|V^{-}(S)\right| \in\{0,1,2\}$. Let $\mathcal{Q}_{i}$ collect all subsets of these sets that belong to $i$ 's neighborhood, i.e. $\mathcal{Q}_{i}:=\left\{Q \subseteq V \mid Q=\left(V_{i} \cup i\right) \cap S\right.$ for some $\left.S \in \mathcal{S}_{i}\right\}$. A network is called "weakly balanced" if for every voter $i \in V$ and for every $Q \in \mathcal{Q}_{i}$, there is a corresponding set of agents $S$ with $Q \subseteq S \in \mathcal{S}_{i}$, which is weakly better informed than the complementary set, i.e. $\prod_{j \in S} \frac{p_{j}}{1-p_{j}} \geq \prod_{k \in V \backslash S} \frac{p_{k}}{1-p_{k}}$.

Strong balancedness requires that any group of voters $S$ which is better informed than the complementary set is also considered as better informed by a majority of voters. Weak balancedness addresses groups of voters $Q$ within a given voter's neighborhood, which together with some voters outside of the neighborhood would have slightly more believers than non-believers. When the agent is a believer of such a group $Q$, there must be one corresponding group of voters outside the agent's neighborhood such that both groups together are weakly better informed than the complementary set.

Proposition A.2. The sincere strategy profile $\hat{\sigma}$ is efficient if and only if the network is strongly balanced. The sincere strategy profile $\hat{\sigma}$ is an equilibrium if (a) the network is strongly balanced and only if $(b)$ the network is weakly balanced.

Proof. We begin with strong balancedness and then turn to weak balancedness.

Strong balancedness. We first show equivalence between strong balancedness and efficiency of $\hat{\sigma}$.

For a given draw of nature denote by $S$ the set of experts who have received signal $A^{*}$. Generally, a strategy profile is efficient if and only if the outcome is $A$ whenever $A$ is more likely to be the true state than $B$, i.e., whenever

$$
\sum_{j \in S} \log \left(\frac{p_{j}}{1-p_{j}}\right)>\sum_{k \in V \backslash S} \log \left(\frac{p_{k}}{1-p_{k}}\right)
$$

and the outcome is $B$ whenever inequality (A.5) is reversed (e.g., Shapley and Grofman, 1984, Theorem II). Now, consider that under $\hat{\sigma}$ an agent $i$ votes $A$ if and only if he is a believer of the group $S$ who have received signal $A^{*}$, i.e. $i \in V^{+}(S)$. Indeed, a voter $i \in V$ votes $A$ if and only if $A$ has the higher posterior probability to be the true state of the world given $i^{\prime} s$ information, which is $\sum_{j \in\left(V_{i} \cup i\right) \cap S} \log \left(\frac{p_{j}}{1-p_{j}}\right)>\sum_{k \in\left(V_{i} \cup i\right) \backslash S} \log \left(\frac{p_{k}}{1-p_{k}}\right)$, which is the definition of $i \in V^{+}(S)$. Hence, the number of $A$ votes is $\left|V^{+}(S)\right|$, while the number of $B$ votes is $\left|V^{-}(S)\right|$ (again, for the draw of nature that gives signal $A^{*}$ to $S$ and signal $B^{*}$ to $\left.V \backslash S\right)$.

If inequality (A.5) holds, then strong balancedness implies $\left|V^{+}(S)\right|>\left|V^{-}(S)\right|$ such that $A$ receives a majority of votes. If inequality (A.4) is reversed, then by strong balancedness $B$ receives a majority of votes. If the RHS and LHS of inequality (A.5) are equal, then both states of the world are equally likely and any outcome is consistent with efficiency. Hence, strong balancedness implies efficiency of $\hat{\sigma}$.

Now, suppose that $g$ is not strongly balanced. Then there is a set $S \subseteq V$ with $\sum_{i \in S} \log \left(\frac{p_{i}}{1-p_{i}}\right)>\sum_{j \in V \backslash S} \log \left(\frac{p_{j}}{1-p_{j}}\right)$, but $\left|V^{+}(S)\right| \leq\left|V^{-}(S)\right|$. Suppose all $i \in S$ receive signal $A^{*}$ and all $j \in V \backslash S$ receive signal $B^{*}$. Then $\hat{\sigma}$ leads to outcome $B$ or to a tie, while $A$ is more likely to be true. Hence, efficiency of $\hat{\sigma}$ requires strong balancedness. (We have established that $\hat{\sigma}$ is efficient if and only if the network is strongly balanced.)

\footnotetext{
59 Applying the corresponding upcoming Definition A.1 to the specific set-up of section 2 leads to a notion of balancedness that is equivalent to Definition 2.2. This is shown in SOM B.3.
} 
Efficiency of a strategy profile implies that it is an equilibrium, since every player's expected utility is maximal.

Weak balancedness. Suppose weak balancedness is violated, i.e., there is a voter $i \in V$ and a set $Q \in \mathcal{Q}_{i}$, such that there is no corresponding set of agents $S$ with $Q \subseteq S \in \mathcal{S}_{i}$ and $\prod_{j \in S} \frac{p_{j}}{1-p_{j}} \geq \prod_{k \in V \backslash S} \frac{p_{k}}{1-p_{k}}$, i.e., which is weakly better informed than the complementary set. Then $\forall S \in \mathcal{S}_{i}$, we have $\sum_{j \in S} \log \left(\frac{p_{j}}{1-p_{j}}\right)<\sum_{k \in V \backslash S} \log \left(\frac{p_{k}}{1-p_{k}}\right)$ and $\mathcal{S}_{i} \neq \emptyset$ because $\mathcal{Q}_{i} \neq \emptyset$ by assumption.

Consider a draw of nature such that within $i$ 's neighborhood all voters in $Q$ have received signal $A^{*}$ and all others $\left(V_{i} \cup i\right) \backslash Q$ have received signal $B^{*}$. Since $i \in V^{+}(S)$ for $Q \subseteq S \in \mathcal{S}_{i}$, it also holds that $i \in V^{+}(Q)$, and $i$ will vote for $A$ under $\hat{\sigma}$.

Consider the deviation of $i$ to vote $B$ in this case (i.e., when from his own signal and the messages of $V_{i}, i$ infers that within $\left(V_{i} \cup i\right)$ exactly subset $Q$ has received signal $\left.A^{*}\right)$. Let $X$ denote the set of other agents $j \in V \backslash\left(V_{i} \cup i\right)$ who have received signal $A^{*}$. If $(Q \cup X) \notin \mathcal{S}_{i}$, then the deviation has not affected the outcome since it is not the case that there is a slight majority for alternative $A$ under $\hat{\sigma}$. If $(Q \cup X) \in \mathcal{S}_{i}$, then the deviation has turned the outcome from $A$ to $B$, or from $A$ to a tie, or from a tie to $B$. This improves expected utility if the probability that $B$ is the true state is larger than that $A$ is true. By the property that $\forall S \in \mathcal{S}_{i}$, we have $\sum_{j \in S} \log \left(\frac{p_{j}}{1-p_{j}}\right)<\sum_{k \in V \backslash S} \log \left(\frac{p_{k}}{1-p_{k}}\right), B$ is indeed more likely to be true than $A$. Hence, when weak balancedness is violated there is a beneficial deviation from $\hat{\sigma}$.

To interpret Proposition A.2 part (a), consider first the special case of homogenous signal precision $p_{i}=p$ for all vot-

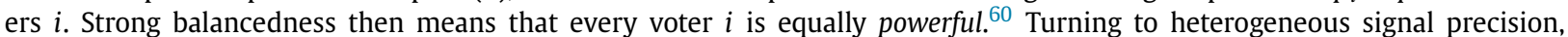
strong balancedness means that heterogeneity in expertise is matched by the heterogeneity in power. For instance, as we have discussed, in Example 2, the star network, as well as in Example 3, sincere voting is not efficient for homogeneous signal precision. However, in Example 2 sincere behavior $\hat{\sigma}$ would be efficient if signal precisions were, e.g., $\left(p_{1}, \ldots, p_{9}\right)=(.9, .6, .6, .6, .6, .5, .5, .5, .5)$. Similarly, in Example 3 , sincere behavior would be efficient if, e.g., signal precisions were $\left(p_{1}, \ldots, p_{9}\right)=(.9, .9, .6, .6 ., .6, .5, .5, .5, .5)$. Hence strong balancedness does not generally refer to an equality of power, but rather to a balance of power with expertise. ${ }^{61}$

To interpret Proposition A.2 part (b), consider an agent $i$ who observes message $A$ from all agents in a set $Q$, of which $i$ is a believer. Sincere behavior is to follow this message. Deviations from sincere behavior only affect the outcome when the number of $A$ votes under $\hat{\sigma}$ is slightly larger than the number of $B$ votes. Suppose that in any of those cases $A$ is less likely to be true than $B$. Then $i$ can beneficially deviate from $\hat{\sigma}$ by voting $B$. We have constructed a violation of weak balancedness and argued that then $\hat{\sigma}$ is not an equilibrium. In fact, the agent $i$ in the example is "cursed" in the sense that when he follows the vote recommendations of the set $Q$ whenever his vote has an effect, it has the undesirable effect of switching the outcome to the less likely state.

This is illustrated by the following example.

Example 4 (not weakly balanced). Let $V=\left\{i_{1}, i_{2}, \ldots, i_{9}\right\}$, the network $g$ as illustrated in Fig. 9, and let the signal precision of all agents $i$ be equal $p_{i}=p>\frac{1}{2}$. This network violates weak balancedness: Consider agent $i_{1}$ and set $Q=\left\{i_{2}, i_{3}\right\}$. Agent $i_{1}$ is a believer of set $Q$ since he assigns more expertise to this set $\left(\left(V_{1} \cup i\right) \cap Q=\left\{i_{2}, i_{3}\right\}\right)$ than to the complementary set, of which he can only observe himself $\left(\left(V_{1} \cup i\right) \backslash Q=\left\{i_{1}\right\}\right)$. There are many elements in $\mathcal{S}_{1}$. For instance, $Q$ together with $X=\left\{i_{8}, i_{9}\right\}$ forms a set $S=\left\{i_{2}, i_{3}, i_{8}, i_{9}\right\} \in \mathcal{S}_{1}$, which indeed has slightly more believers than non-believers since there are five believers $\left(\left\{i_{1}, i_{2}, i_{3}, i_{8}, i_{9}\right\}\right)$ and four non-believers $\left(\left\{i_{4}, i_{5}, i_{6}, i_{7}\right\}\right)$. Other examples that contain set $Q$ are $S^{\prime}=\left\{i_{2}, i_{3}, i_{8}, i_{4}\right\} \in \mathcal{S}_{1}$, or $S^{\prime \prime}=\left\{i_{2}, i_{3}, i_{4}, i_{5}\right\} \in \mathcal{S}_{1}$. Indeed, each of these sets has slightly more believers than non-believers. However, there is no extension of $Q$ in $\mathcal{S}_{1}$, which contains more than four players. Weak balancedness would require that at least one $S$ with $Q \subseteq S \in \mathcal{S}_{1}$ is better informed than the complementary set, but this is not true when only a minority of informed agents are in S. Thus, by Proposition A.2 part (b), the sincere strategy profile $\hat{\sigma}$ is not an equilibrium.

In the example, agent $i_{1}$ has an incentive to deviate from the sincere strategy profile when he infers from their messages that the agents $Q=\left\{i_{2}, i_{3}\right\}$ have received, e.g., signal $A^{*}$, while he has received signal $B^{*}$. Given this private information, $A$ is clearly more likely to be true than $B$ since two out of three "observable" voters have received signal $A^{*}$. However, conditioning on pivotality implies that at least five voters (e.g. $\left.\left\{i_{1}, i_{4}, i_{5}, i_{6}, i_{7}\right\}\right)$ must have received signal $B^{*}$. Thus, in this situation the unconditional posterior of voter $i_{1}$ differs crucially from the posterior conditional on pivotality, which is the reason for the "swing voter's curse." The source of this curse is the power of agents $i_{2}$ and $i_{3}$ regarding the votes of agents $i_{1}, i_{8}$, and $i_{9}$.

More generally, suppose there is an agent who observes a set of agents $Q$ sending the same message, say $A$. If for any additional set of agents whose votes for $A$ would render $i$ pivotal (call this set $X$ ), it holds that these two sets together are less well-informed than the complementary set $V \backslash(Q \cup X)$, then voting for $A$ is not a best response.

\footnotetext{
$\overline{60}$ This result is shown as Proposition B.6 in SOM B.4. Power is defined as the Shapley-Shubik index or the Banzhaf index in a cooperative voting game that incorporates how many believers each coalition has. SOM B.4 introduces this framework, provides the result, and also gives some intuition for how individual power is determined by the network structure.

61 With our experimental design we purposefully keep expertise constant among experts to observe unbalanced power directly in the network structure.
} 


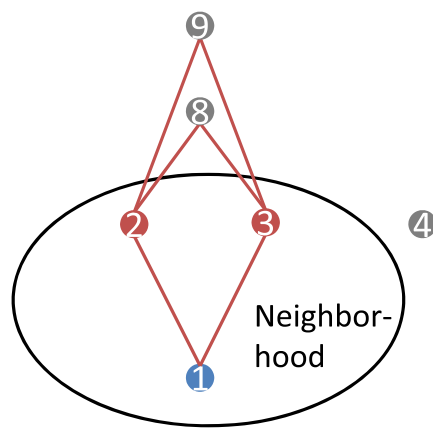

Fig. 9. Example 4, a network in which weak balancedness is violated. Voter $i_{1}$ is "cursed" if his signal differs from the signals of voters $i_{2}$ and $i_{3}$.

Inefficient equilibria revisited. Turning to Proposition 2.3, the existence of inefficient equilibria with information transmission trivially extends to the more general framework. Thus, there are network structures and distributions of expertise that lead to inefficient equilibria.

Proposition A.3. There are networks in which the sincere strategy profile $\hat{\sigma}$ is both an equilibrium and exhibits informational inefficiency. This inefficiency does not necessarily vanish when the number of experts grows large.

Proof. We show existence of inefficient strategy profiles with the network introduced in Example 3 . For any $t=1,2, \ldots$ we consider a network with two experts of degree $2 t, 1+2 t$ experts of degree zero and $4 t$ non-experts of degree one. For $t=1$ this is exactly the network depicted in Fig. 2. All experts have signal quality $p_{j}=p>0.5$, all non-experts signal quality $p_{i}=0.5$. For any $t=1,2, \ldots$, denote the corresponding game by $\Gamma^{t}$ and the sincere strategy profile in that game by $\hat{\sigma}^{t}$.

Under $\hat{\sigma}^{t}, 3+6 t$ agents participate in the vote. If the two senders receive the same signal, say $A^{*}$, then $A$ is the outcome since the two senders induce $2 *(1+2 t) \geq 2+3 t A$-votes. If both senders receive different signals, $A^{*}$ and $B^{*}$, then $A$ wins if and only if $A$ receives $k \geq 1+t$ votes of the $1+2 t$ experts with degree zero. Supposing that $A$ is the true state, the probability that the outcome is $A$ provides the general probability that the outcome coincides with the true state since $\hat{\sigma}^{t}$ treats $A$ and $B$ interchangeably. Thus, under $\hat{\sigma}^{t}$ the probability that the outcome coincides with the true state is

$$
E U\left(\hat{\sigma}^{t}\right)=p^{2} * 1+2 p(1-p) \sum_{k=t+1}^{2 t+1}\left(\begin{array}{c}
2 t+1 \\
k
\end{array}\right) p^{k}(1-p)^{2 t+1-k}+(1-p)^{2} * 0 .
$$

Inefficiency. We establish inefficiency of $\hat{\sigma}^{t}$ for any $t$ and also in the limit. (Recall that a strategy profile is efficient if and only if for any draw of nature it selects the outcome that maximizes the probability to match the true state.) Consider the draw of nature in which both senders receive signal $A^{*}$ and all other experts receive signal $B^{*}$. An efficient strategy profile would implement (the majority signal) $B^{*}$, but $\hat{\sigma}^{t}$ leads to $A$.

For an efficient strategy profile $\sigma^{t}$ the probability that the outcome coincides with the true state is below one for finite $t$, but converges to one for growing $t$, i.e. $\lim _{t \rightarrow \infty} E U\left(\sigma^{t}\right)=1$ when $\sigma^{t}$ efficient. Under $\hat{\sigma}^{t}$, when both senders happen to receive the incorrect signal, then the outcome does not coincide with the true state. Thus, the probability of implementing the incorrect outcome under $\hat{\sigma}^{t}$ is at least $(1-p)^{2}$, which is independent of $t$. Hence, $\lim _{t \rightarrow \infty} E U\left(\hat{\sigma}^{t}\right) \leq 1-(1-p)^{2}<1$, i.e., inefficiency does not vanish for growing $t$.

Now, we establish that $\hat{\sigma}^{t}$ is an equilibrium for any $t$. We show first that there is no profitable deviation that occurs on the voting stage only. Then we show that there is no profitable deviation that affects both stages voting and communication.

Deviations on the voting stage only. Consider a voter $i \in V$ who considers to deviate from $\hat{\sigma}^{t}$ by changing his voting strategy $v_{i}$. This can be a non-expert who does not follow the received message or an expert who does not vote the received signal, but chooses some different strategy instead.

Suppose one sender (i.e., a voter with $p_{j}=p>0.5$ and $d_{j}=2 t$ ) receives signal $A^{*}$ and the other sender receives signal $B^{*}$. Then $A$ receives more votes than $B$ under $\hat{\sigma}^{t}$ if and only if more experts with degree zero (i.e., voters with $p_{j}=p>0.5$ and $d_{j}=0$ ) have received signal $A^{*}$. Hence, when the two senders have not received the same signal, then $\hat{\sigma}^{t}$ always implements the majority signal and thus induces the outcome that is more likely to be true. Hence, if there is a beneficial deviation, then it must also change outcomes in which both senders have received the same signal.

Suppose that both senders have received the same signal, say $A^{*}$. Then the number of $A$-votes under $\hat{\sigma}^{t}$ is at least $2+4 t$ ( since two senders, and $2 * 2 t$ non-experts vote for $A$ ) and the number of $B$-votes is hence at most $3+6 t-(2+4 t)=1+2 t$. The number of $A$-votes thus exceeds the number of $B$-votes by at least $2+4 t-(1+2 t)=1+2 t \geq 3$ votes. Hence, a single agent who changes her vote cannot affect the outcome if the two senders have received the same signal.

Taken together a deviation that only changes one vote is neither beneficial if both senders have received the same signal nor if they have received different signals. This precludes deviation incentives of non-experts, of experts with degree zero, 
as well as of senders who consider to deviate in their voting behavior only, i.e., all deviations that happen on the voting stage only. We now turn to deviations that also affect the communication stage, i.e., which involve a sender who does not truthfully transmit her signal, and show that any of those is neither beneficial. ${ }^{62}$

Deviations on both stages. Consider a sender $j \in V$ with $d_{j}>0$. This expert has $(3 \times 3)^{2}=81$ strategies because she chooses one of three messages and one of three voting actions after receiving one of two signals. ${ }^{63}$ To evaluate different strategies we can assume w.l.o.g. that the expert has received signal $A^{*}$ because neither the utility function nor the strategy profile depends on the label of the alternatives. This reduces the number of strategies to the following nine: $\left(m_{j}\left(A^{*}\right), v_{j}\left(A^{*}\right)\right) \in\{(A, A),(A, B),(A, \emptyset),(B, A),(B, B),(B, \emptyset),(\emptyset, A),(\emptyset, B),(\emptyset, \emptyset)\}$. The first strategy $(A, A)$ is sincere and hence not a deviation. The strategies $(A, B)$ and $(A, \emptyset)$ only involve deviations on the voting stage and are hence not beneficial by the paragraph above. This leads to the following six remaining deviations $\tilde{\sigma}$ and their corresponding expected utilities $E U\left(\tilde{\sigma}^{t}\right)^{64}$ :

1. Sender $j$ sends the opposite message and votes the signal.

$$
E U\left(\tilde{\sigma}^{t}\right)=p^{2} \sum_{k=t}^{2 t+1}\left(\begin{array}{c}
2 t+1 \\
k
\end{array}\right) p^{k}(1-p)^{2 t+1-k}+p(1-p)+(1-p)^{2} \sum_{k=t+2}^{2 t+1}\left(\begin{array}{c}
2 t+1 \\
k
\end{array}\right) p^{k}(1-p)
$$

2. Sender $j$ sends the opposite message and votes the opposite.

$$
E U\left(\tilde{\sigma}^{t}\right)=\left[p^{2}+(1-p)^{2}\right] \sum_{k=t+1}^{2 t+1}\left(\begin{array}{c}
2 t+1 \\
k
\end{array}\right) p^{k}(1-p)^{2 t+1-k}+p(1-p)
$$

3. Sender $j$ sends the opposite message and abstains.

$$
\begin{aligned}
& E U\left(\tilde{\sigma}^{t}\right)=p^{2}\left[\frac{1}{2}\left(\begin{array}{c}
2 t+1 \\
t
\end{array}\right) p^{t}(1-p)^{t+1}+\sum_{k=t+1}^{2 t+1}\left(\begin{array}{c}
2 t+1 \\
k
\end{array}\right) p^{k}(1-p)^{2 t+1-k}\right] \\
& +p(1-p)+(1-p)^{2}\left[\frac{1}{2}\left(\begin{array}{c}
2 t+1 \\
t+1
\end{array}\right) p^{t+1}(1-p)^{t}+\sum_{k=t+2}^{2 t+1}\left(\begin{array}{c}
2 t+1 \\
k
\end{array}\right) p^{k}(1-p)^{2 t+1-k}\right]
\end{aligned}
$$

4. Sender $j$ sends the empty message and votes the signal.

$$
E U\left(\tilde{\sigma}^{t}\right)=p^{2}+p(1-p) p^{2 t+1}+p(1-p) \sum_{k=1}^{2 t+1}\left(\begin{array}{c}
2 t+1 \\
k
\end{array}\right) p^{k}(1-p)^{2 t+1-k}
$$

5. Sender $j$ sends the empty message and votes the opposite.

$$
E U\left(\tilde{\sigma}^{t}\right)=p^{2} \sum_{k=1}^{2 t+1}\left(\begin{array}{c}
2 t+1 \\
k
\end{array}\right) p^{k}(1-p)^{2 t+1-k}+p(1-p)+(1-p)^{2} p^{2 t+1}
$$

6. Sender $j$ sends the empty message and abstains.

$$
E U\left(\tilde{\sigma}^{t}\right)=p^{2}\left[1-\frac{1}{2}(1-p)^{2 t+1}\right]+p(1-p) \frac{1}{2} p^{2 t+1}+p(1-p)\left[1-\frac{1}{2}(1-p)^{2 t+1}\right]+(1-p)^{2} \frac{1}{2} p^{2 t+1}
$$

The derivation of the expressions (A.7)-(A.12) is shown in SOM B.5. We can now compare the expected utility $E U\left(\tilde{\sigma}^{t}\right)$ of each deviation, which is given by (A.7)-(A.12), with the expected utility of the sincere strategy profile $E U\left(\hat{\sigma}^{t}\right)$, which is given by (A.6).

\footnotetext{
62 For large $t$ this is simple to show. In the case in which the deviating agent receives the correct signal, say $A^{*}$, and the other sender receives the incorrect signal, the probability that the outcome is $A$ approaches zero for growing $t$. Hence, the expected utility of any such deviation is bounded from above by $\lim _{t \rightarrow \infty} E U\left(\tilde{\sigma}^{t}\right) \leq 1-p(1-p)<1-(1-p)^{2}=\lim _{t \rightarrow \infty} E U\left(\hat{\sigma}^{t}\right)$.

${ }^{63}$ In general, voters with positive degree $d_{i}>0$ have more pure strategies. In this example, the senders are linked to non-experts (i.e. voters $i$ with $\left.p_{i}=0.5\right)$ who are assumed by convention not to send a message under $\hat{\sigma}^{t}$. Since a message of an uninformed voter is meaningless, a change of convention would not affect the result.

64 Deviations that involve to vote and/or communicate an alternative unconditionally, i.e., independent of the signal, need not be considered here because of the symmetry between the alternatives. Indeed, if it is beneficial to vote $B$ after receiving $A^{*}$, then it is also beneficial to vote $A$ after receiving $B^{*}$, which is to vote the opposite of the signal. Similarly, there is no need to consider strategies that involve the empty message and/or to abstain only after one of the two signals. Indeed, if it is beneficial e.g. to abstain after having received signal $A^{*}$, then it is also beneficial to abstain after having received signal $B^{*}$, which is to abstain unconditionally. Hence, if none of the six symmetric deviations is an improvement over $\hat{\sigma}^{t}$, then neither is a deviation that treats the alternatives $A$ and $B$ asymmetrically.
} 
Consider, for instance, the fifth deviation: Sender $j$ sends the empty message and votes the opposite of the signal. There are $3+4 t$ votes and $2+2 t$ is a majority. Denote by $\left(s_{j}, s_{k}\right)$ the signals of the two senders. There are four possibilities.

- $\left(A^{*}, A^{*}\right): A$ wins if there are at least $2+2 t-(1+2 t)=1 A^{*}$-signals among the experts of degree zero.

- $\left(A^{*}, B^{*}\right): A$ never wins since $B$ receives at least $2+2 t$ votes.

- $\left(B^{*}, A^{*}\right): A$ wins since it receives at least $2+2 t$ votes.

- $\left(B^{*}, B^{*}\right)$ : $A$ wins if there are at least $2+2 t-1=2 t+1 A^{*}$-signals among the experts of degree zero, i.e., all of them have signal $A^{*}$.

We now show that this deviation is not beneficial by considering the change in expert $j$ 's expected utility (which is the expected utility of every agent). Supposing that the true state is $A$, the expected utility is the likelihood that $A$ is indeed implemented. Hence,

$$
E U\left(\tilde{\sigma}^{t}\right)=p^{2} \sum_{k=1}^{2 t+1}\left(\begin{array}{c}
2 t+1 \\
k
\end{array}\right) p^{k}(1-p)^{2 t+1-k}+p(1-p) * 0+p(1-p) * 1+(1-p)^{2} p^{2 t+1},
$$

which directly simplifies to (A.11).

For the upcoming simplifications we use the following two properties:

1. $\sum_{k=0}^{2 t+1}\left(\begin{array}{c}2 t+1 \\ k\end{array}\right) p^{k}(1-p)^{2 t+1-k}=1$ and

2. $\left(\begin{array}{c}2 t+1 \\ k\end{array}\right)=\left(\begin{array}{c}2 t+1 \\ 2 t+1-k\end{array}\right)$ for any $k=0, \ldots, 2 t+1$.

Let $\Delta:=E U\left(\hat{\sigma}^{t}\right)-E U\left(\tilde{\sigma}^{t}\right)$. Then

$$
\begin{aligned}
\Delta= & p^{2}\left[1-\sum_{k=1}^{2 t+1}(\ldots)\right]+p(1-p)\left[2 \sum_{k=t+1}^{2 t+1}\left(\begin{array}{c}
2 t+1 \\
k
\end{array}\right) p^{k}(1-p)^{2 t+1-k}-1\right]-(1-p)^{2} p^{2 t+1} \\
\Delta= & p^{2}\left[\sum_{k=0}^{2 t+1}(\ldots)-\sum_{k=1}^{2 t+1}(\ldots)\right]+p(1-p)\left[2 \sum_{k=t+1}^{2 t+1}\left(\begin{array}{c}
2 t+1 \\
k
\end{array}\right) p^{k}(1-p)^{2 t+1-k}-1\right] \\
& \left.-(1-p)^{2} p^{2 t+1}\right] \\
\Delta= & p^{2}(1-p)^{2 t+1}+p(1-p) \underbrace{\left[\sum_{k=t+1}^{2 t+1}(\ldots)-\sum_{k=0}^{2 t+1}(\ldots)\right.}_{=-\sum_{k=0}^{t}(\ldots)}]+\underbrace{p(1-p) \sum_{k=t+1}^{2 t+1}(\ldots)-(1-p)^{2} p^{2 t+1}}_{\geq p(1-p) \sum_{k=t+1}^{2 t}(\ldots)}
\end{aligned}
$$

To simplify the last part of the equation notice the following:

- First, $\sum_{k=t+1}^{2 t+1}\left(p^{k}(1-p)^{2 t+1-k}=\sum_{k=t}^{2 t}\left(p^{k}(1-p)^{2 t+1-k}+\left(\begin{array}{c}2 t+1 \\ 2 t+1\end{array}\right) p^{2 t+1}(1-p)^{0}\right.\right.$.

- Second, $\left(\begin{array}{c}2 t+1 \\ 2 t+1\end{array}\right) p^{2 t+1}(1-p)^{0}=p^{2 t+1}$.

- Third, $p(1-p) p^{2 t+1}-(1-p)^{2} p^{2 t+1}=\left[p(1-p)-\left(1-p^{2}\right)\right] p^{2 t+1} \geq 0$.

Thus,

$$
\begin{aligned}
& \Delta \geq p^{2}(1-p)^{2 t+1}-p(1-p) \sum_{k=0}^{t}\left(\begin{array}{c}
2 t+1 \\
k
\end{array}\right) p^{k}(1-p)^{2 t+1-k}+p(1-p) \sum_{k=t+1}^{2 t}(\ldots) \\
& \Delta \geq \underbrace{p^{2}(1-p)^{2 t+1}-p(1-p)\left(\begin{array}{c}
2 t+1 \\
0
\end{array}\right) p^{0}(1-p)^{2 t+1}-p(1-p) \sum_{k=1}^{t}(\ldots)+p(1-p) \sum_{k=t+1}^{2 t}(\ldots)}_{\geq 0} \\
& \Delta \geq \underbrace{p(1-p)}_{\geq 0}\left[\sum_{k=t+1}^{2 t}\left(\begin{array}{c}
2 t+1 \\
k
\end{array}\right) p^{k}(1-p)^{2 t+1-k}-\sum_{k=1}^{t}\left(\begin{array}{c}
2 t+1 \\
k
\end{array}\right) p^{k}(1-p)^{2 t+1-k}\right]
\end{aligned}
$$

Hence, $\Delta \geq 0$ if 


$$
\sum_{k=t+1}^{2 t}\left(\begin{array}{c}
2 t+1 \\
k
\end{array}\right) p^{k}(1-p)^{2 t+1-k} \geq \sum_{k=1}^{t}\left(\begin{array}{c}
2 t+1 \\
k
\end{array}\right) p^{k}(1-p)^{2 t+1-k}
$$

To show that inequality (A.13) holds, we substitute $k$ in the first sum by $l \equiv 2 t+1-k$ and consistently sum over $l=1, \ldots, t$ (instead over $k=t+1, \ldots, 2 t)$. Moreover, we use $\left(\begin{array}{c}2 t+1 \\ k\end{array}\right)=\left(\begin{array}{c}2 t+1 \\ 2 t+1-k\end{array}\right)$.

$$
\begin{aligned}
\sum_{l=1}^{t}\left(\begin{array}{c}
2 t+1 \\
l
\end{array}\right) p^{2 t+1-l}(1-p)^{l}-\sum_{k=1}^{t}\left(\begin{array}{c}
2 t+1 \\
k
\end{array}\right) p^{k}(1-p)^{2 t+1-k} \geq 0 \\
\sum_{l=1}^{t}\left(\begin{array}{c}
2 t+1 \\
l
\end{array}\right)\left(p^{2 t+1-l}(1-p)^{l}-p^{l}(1-p)^{2 t+1-l}\right) \geq 0
\end{aligned}
$$

For every $l=1, \ldots, t$, we have $2 t+1-l>l$. This implies for the expression in brackets that the first product $\left(p^{2 t+1-l}(1-p)^{l}\right)$ is larger than the second product $\left(p^{l}(1-p)^{2 t+1-l}\right)$. Hence, the inequality above holds, which implies inequality (A.13). Thus, $E U\left(\hat{\sigma}^{t}\right) \geq E U\left(\tilde{\sigma}^{t}\right)$ and hence this deviation $\tilde{\sigma}^{t}$ is not beneficial.

Using the same techniques as for this deviation, we can show for all six deviations $\tilde{\sigma}^{t}$ that $E U\left(\tilde{\sigma}^{t}\right) \leq E U\left(\hat{\sigma}^{t}\right){ }^{65}$ Hence, no deviation that involves both stages communication and voting is profitable either.

\section{A.2.4. Multiplicity}

In the sincere strategy profile $\hat{\sigma}$, all communication channels, i.e., links in $g$, are used. Generally, the information transmission network $g^{*}$ under some strategy profile $\sigma$ need not coincide with the exogenous network $g$, but can be any subnetwork ( $g^{*} \subseteq g$ ) of it, which uses some but not necessarily all of the given communication channels (cf. SOM B.2). For instance, any network $g$ that contains a subnetwork $g^{\prime} \subseteq g$ which satisfies strong balancedness admits an efficient equilibrium by using the subnetwork as communication network, i.e., $g^{*}=g^{\prime}$. Our model extension admits denser networks $g$ and hence gives rise to many more information transmission networks $g^{*} \subseteq g$ than our baseline model. As a consequence, coordination on an efficient equilibrium might become even harder than in the baseline model.

Private versus public communication. With overlapping audiences, we can not only model private communication but also public communication. Communication is fully public if the network $g$ is complete $\left(g=g^{V}\right)$, i.e., every voter is linked to every other voter. In that case, the sincere strategy profile $\hat{\sigma}$ is efficient and an equilibrium. In this deliberation equilibrium the optimal alternative can be deduced by every voter such that this information aggregation within each individual determines votes unanimously (and other voting rules than the majority rule would also admit a similar equilibrium, cf. Gerardi and Yariv, 2007). More generally, in every network $g$, in which a non-empty subset $S$ of voters is linked to all informed voters, there are efficient equilibria in which the members of $S$ vote for the more likely alternative. This is how public communication admits efficient information aggregation in the communication stage.

At the other extreme of the spectrum, communication can be fully private as in the model studied in section 2, i.e., when the network $g$ is bipartite and voters in one group (the non-experts) have at most degree one. There each voter holds at most one piece of information after communication such that information aggregation can only occur in the voting stage. Arguably, in reality communication is neither fully public nor fully private. Receiving multiple messages and an own signal leads to information aggregation already in the communication stage. Participation in a majority election further aggregates information in the voting stage. Whether such institutions are efficient depends on the balancedness of the social network.

\section{Appendices B and C. Supplementary online material (SOM)}

Supplementary online material (SOM) related to this article can be found online at https://doi.org/10.1016/j.geb.2019.08. 009.

\section{References}

Agranov, M., Goeree, J.K., Romero, J., Yariv, L., 2017. What makes voters turn out: the effects of polls and beliefs. J. Eur. Econ. Assoc. 16, 825-856.

Austen-Smith, D., Banks, J.S., 1996. Information aggregation, rationality, and the Condorcet jury theorem. Am. Polit. Sci. Rev. 90, 34-45.

Austen-Smith, D., Feddersen, T.J., 2006. Deliberation, preference uncertainty, and voting rules. Am. Polit. Sci. Rev. 100, $209-217$.

Battaglini, M., 2017. Public protests and policy making. Q. J. Econ. 132, 485-549.

Battaglini, M., Morton, R.B., Palfrey, T.R., 2010. The swing voter's curse in the laboratory. Rev. Econ. Stud. 77, 61-89.

Bock, O., Baetge, I., Nicklisch, A., 2014. hroot: Hamburg registration and organization online tool. Eur. Econ. Rev. 71, 117-120.

Buechel, B., Mechtenberg, L., 2017. The swing voter's curse in social networks, Working Paper, http://doc.rero.ch/record/305039.

Carpini, M.X.D., Cook, F.L., Jacobs, L.R., 2004. Public deliberation, discursive participation, and citizen engagement: a review of the empirical literature. Annu. Rev. Pol. Sci. 7, 315-344.

M.d. Condorcet, Marie Jean Antoine Nicolas de Caritat, 1785. Essai sur l'application de l'analyse à la probabilité des décisions rendues à la pluralité des voix. L'imprimerie royale.

$\overline{65}$ These derivations can be found in SOM B.5. 
Coughlan, P.J., 2000. In defense of unanimous jury verdicts: mistrials, communication, and strategic voting. Am. Polit. Sci. Rev. 94, 375-393.

Crawford, V.P., Sobel, J., 1982. Strategic information transmission. Econometrica, 1431-1451.

DeMarzo, P.M., Vayanos, D., Zwiebel, J., 2003. Persuasion bias, social influence, and unidimensional opinions. Q. J. Econ. 118, 909-968.

Dittmann, I., Kübler, D., Maug, E., Mechtenberg, L., 2014. Why votes have value: instrumental voting with overconfidence and overestimation of others' errors. Games Econ. Behav. 84, 17-38.

Elbittar, A., Gomberg, A., Martinelli, C., Palfrey, T.R., 2016. Ignorance and bias in collective decisions. J. Econ. Behav. Organ.

Enke, B., Zimmermann, F., 2017. Correlation neglect in belief formation. Rev. Econ. Stud. 86, 313-332.

Esponda, I., Vespa, E., 2014. Hypothetical thinking and information extraction in the laboratory. Am. Econ. J. Microecon. 6, 180-202.

Feddersen, T., Pesendorfer, W., 1996. The swing voter's curse. Am. Econ. Rev., 408-424.

Feddersen, T., Pesendorfer, W., 1997. Voting behavior and information aggregation in elections with private information. Econometrica, 1029-1058.

Feddersen, T., Pesendorfer, W., 1998. Convicting the innocent: the inferiority of unanimous jury verdicts under strategic voting. Am. Polit. Sci. Rev., 23-35.

Gerardi, D., Yariv, L., 2007. Deliberative voting. J. Econ. Theory 134, 317-338.

Goeree, J.K., Yariv, L., 2011. An experimental study of collective deliberation. Econometrica 79, 893-921.

Golub, B., Jackson, M.O., 2010. Naïve learning in social networks and the wisdom of crowds. Am. Econ. J. Microecon. 2, $112-149$.

Golub, B., Jackson, M.O., 2012. How homophily affects the speed of learning and best-response dynamics. Q. J. Econ. 127, $1287-1338$.

Grosser, J., Seebauer, M., 2016. The curse of uninformed voting: an experimental study. Games Econ. Behav. 97, 205-226.

Guarnaschelli, S., McKelvey, R.D., Palfrey, T.R., 2000. An experimental study of jury decision rules. Am. Polit. Sci. Rev. 94, 407-423.

Iaryczower, M., Shi, X., Shum, M., 2018. Can words get in the way? The effect of deliberation in collective decision making. J. Polit. Econ. 126, 688-734.

Jeong, D., 2019. Using cheap talk to polarize or unify a group of decision makers. J. Econ. Theory 180, 50-80.

Kawamura, K., Vlaseros, V., 2017. Expert information and majority decisions. Journal of Public Economics 147, 77-88.

Levy, G., Razin, R., 2015. Correlation neglect, voting behavior, and information aggregation. Am. Econ. Rev. 105, 1634-1645.

Liu, S., 2019. Voting with public information. Games Econ. Behav. 113, 694-719.

Lu, L., Yuan, Y.C., McLeod, P.L., 2012. Twenty-five years of hidden profiles in group decision making a meta-analysis. Personal. Soc. Psychol. Rev. 16, 54-75.

McLennan, A., 1998. Consequences of the Condorcet jury theorem for beneficial information aggregation by rational agents. Am. Polit. Sci. Rev. 92, 413-418.

Morton, R.B., Tyran, J.-R., 2011. Let the experts decide? Asymmetric information, abstention, and coordination in standing committees. Games Econ. Behav. 72, 485-509.

Nitzan, S., Paroush, J., 1982. Optimal decision rules in uncertain dichotomous choice situations. Int. Econ. Rev., 289-297.

Ortoleva, P., Snowberg, E., 2015. Overconfidence in political behavior. Am. Econ. Rev. 105, 504-535.

Palfrey, T.R., Pogorelskiy, K., 2017. Communication among voters benefits the majority party. Econ. J. 129, 961-990.

Pogorelskiy, K., Shum, M., 2019. News We Like to Share: How News Sharing on Social Networks Influences Voting Outcomes. CAGE Online Working Paper Series.

Roth, A.E., 1988. The Shapley Value: Essays in Honor of Lloyd S. Shapley. Cambridge University Press.

Shapley, L., Grofman, B., 1984. Optimizing group judgmental accuracy in the presence of interdependencies. Public Choice 43, 329-343. 